\title{
On Singular Limits of Mean-Field Equations
}

\author{
July 7, 2000 \\ Jean Dolbeault ${ }^{1}$, Peter A. Markowich ${ }^{2}$, \\ Andreas Unterreiter ${ }^{3}$ \\ ${ }^{1}$ CEREMADE, Place du Maréchal de Lattre de Tassigny, \\ F-75775 Paris Cedex 16, France, \\ dolbeault@ceremade.dauphine.fr, \\ http://www.ceremade.dauphine.fr/ ${ }^{\text {dolbeaul/, }}$ \\ ${ }^{2}$ Institut für Mathematik, Universität Wien, \\ A-1090 Wien, Austria, \\ Peter.Markowich@univie.ac.at \\ ${ }^{3}$ Fachbereich Mathematik, Universität Kaiserslautern, \\ D-67653 Kaiserslautern, Germany, \\ unterreiter@mathematik.uni-kl.de, \\ http://www.mathematik.uni-kl.de/ unterreiter.
}

\section{Introduction}

Consider two species of oppositely charged particles (e.g., negatively charged conduction electrons and positively charged holes in a semiconductor crystal or negatively and positively charged ions in a plasma) whose motion is 
described macroscopically be the drift-diffusion-Poisson system

$$
\begin{aligned}
n_{t} & =\operatorname{div}(\nabla f(n)-n \nabla \Phi) \\
p_{t} & =\operatorname{div}(\nabla f(p)+p \nabla \Phi) \\
\lambda \Delta \Phi & =n-p-C(x) .
\end{aligned}
$$

Here $n \geq 0$ and $p \geq 0$ are the position densities of the negatively and, repectively, positively charged particles, $\Phi$ is the (self-consistent) Coulomb potential and $C(x) \in L^{\infty}(\Omega)$ is the difference of background ion position densities. $\lambda>0$ is a (usually small) parameter representing the scaled Debyelength of the particle system. $f=f(s)$ is the (nonlinear) pressure function satisfying $f^{\prime}(s)>0$ for $s>0$. For the sake of simplicity we assume in this paper equal pressure-density equations of state for both particles.

The equations (1),(2),(3) are posed in a bounded domain $\Omega \subset \mathbb{R}^{d}, d \in \mathbb{N}$, where the particles are assumed to be confined. We assume zero-outflux conditions:

$$
(\nabla f(n)-n \nabla \Phi) \cdot \boldsymbol{\nu}=(\nabla f(p)+p \nabla \Phi) \cdot \boldsymbol{\nu}=0 \quad \text { on } \partial \Omega,
$$

and a zero-outward electric field

$$
\nabla \Phi \cdot \boldsymbol{\nu}=0 \quad \text { on } \partial \Omega .
$$

Here $\boldsymbol{\nu}$ denotes the (formal) exterior outward unit vector normal of $\partial \Omega$.

Also we supplement the equations by initial conditions for the densities

$$
\begin{aligned}
& n(x, t=0)=n_{\circ}(x) \geq 0 \quad, \quad \int_{\Omega} n_{\circ}(x) d x=: N \\
& p(x, t=0)=p_{\circ}(x) \geq 0 \quad, \quad \int_{\Omega} p_{\circ}(x) d x=: P
\end{aligned}
$$

$N$ and $P$ are the total negative and, respectively, positive charges, which are conserved by the evolution of (1),(2). Therefore we do have to require total charge neutrality of the system

$$
N-P=\int_{\Omega} C(x) d x .
$$

As basic reference for drift-diffusion-Poisson systems with linear diffusion we cite [MRS90], where many further references can be found. Recently, the 
limit $t \rightarrow \infty$ has been investigated for the bounded domain [Gaj85, GaGr89, CJMTU00, Ott00] and for the whole space problem [BiDo99, BDM99, AMT98, AMTU98, CJMTU00, Ott00] and the limit $\lambda \rightarrow 0$ of the parabolic- elliptic system ws analysed in [Gas, GLMS99] (where results for time intervals of $O(1)$-length were obtained).

In this paper we are interested in the limit $\lambda \rightarrow 0+$ of the stationary system corresponding to (1)-(8). To compute the steady state of a nonlinear (possibly degenerate) convection-diffusion equation with given potential $V=$ $V(x) \in L^{\infty}(\Omega)$,

$$
u_{t}=\operatorname{div}(\nabla f(u)+u \nabla V(x)), \quad \int_{\Omega} u d x=M \geq 0, \quad u \geq 0
$$

we write the flux as

$$
\nabla f(u)+u \nabla V(x)=u \nabla(h(u)+V(x))
$$

where the enthalpy $h=h(s)$ is defined by

$$
h(s)=\int_{1}^{s} \frac{f^{\prime}(r)}{r} d r .
$$

Then for the equilibrium state $u=u_{\infty}(x)$ we find that $h\left(u_{\infty}(x)\right)+V(x)$ is constant on connected components of $\left\{u_{\infty}>0\right\}$. It turns out that the unique equilibrium state, which minimizes the corresponding entropy [CJMTU00], is given by

$$
u_{\infty}(x)=g(c-V(x))
$$

where $g$ is the generalized inverse of the enthalpy,

$$
g(\sigma)=\left\{\begin{array}{cc}
0 & , \quad \sigma \leq \underline{h}:=h(0+) \\
h^{-1}(t) & , \quad \underline{h}<\sigma<\bar{h}:=h(\infty)
\end{array}\right\}, \quad \forall \sigma \in(-\infty, \bar{h}) .
$$

The constant $c$ in (10) is determined such that

$$
M=\int_{\Omega} g(c-V(x)) d x
$$


Note that a unique constant $c$ satisfying (12) exists (at least) for

$$
M \in(0, \bar{M}), \quad \bar{M}=\left\{\begin{array}{cc}
\infty & \text { if } \bar{h}=\infty \\
\int_{\Omega} g\left(\bar{h}+\operatorname{ess} \inf _{\Omega} V-V(x)\right) d x & \text { if } \bar{h}<\infty
\end{array}\right.
$$

For the typical choice

$$
f(s)=s^{m}, \quad \begin{cases}m>1 & : \text { porous medium case } \\ m=1: & \text { linear case } \\ m<1: & \text { fast diffusion case }\end{cases}
$$

we calculate

$$
h(s)=\left\{\begin{array}{cll}
\frac{m}{m-1}\left(s^{m-1}-1\right) & , m>1 & \left(-\infty<\underline{h}=-\frac{m}{m-1}, \bar{h}=\infty\right) \\
\log (s) & , m=1 \quad(-\infty=\underline{h}, \bar{h}=\infty) \\
\frac{m}{1-m}\left(1-s^{-(1-m)}\right) & , m<1 & \left(-\infty=\underline{h}, \bar{h}=\frac{m}{1-m}<\infty\right)
\end{array}\right.
$$

and

$$
g(\sigma)=\left\{\begin{array}{cc}
\left(\left(1+\sigma \frac{m-1}{m}\right)^{+}\right)^{\frac{1}{m-1}} & , m>1 \\
\exp (\sigma) & , m=1 \\
\left(1-\sigma \frac{1-m}{m}\right)^{-\frac{1}{1-m}} & , m<1
\end{array}\right.
$$

Another physically important case is given by the Fermi-Dirac distribution [Groe86, GaGr89]

$$
f(s)=s F^{-1}(s)-\int_{0}^{s} F^{-1}(\xi) d \xi
$$

with

$$
h(s)=F^{-1}(s)-F^{-1}(1), \quad-\infty=\underline{h}, \bar{h}=\infty
$$

and

$$
g(\sigma)=F\left(\sigma+F^{-1}(1)\right)
$$


where

$$
F(\sigma)=\int_{\mathbb{R}^{d}} \frac{d \boldsymbol{v}}{\varepsilon+\exp \left(\frac{|\boldsymbol{v}|^{2}}{2}-\sigma\right)} .
$$

By formally applying the solution formula (10) to (1)-(7), we obtain the steady state drift-diffusion-Poisson system (called the "mean field equation" in the sequel) for the equilibrium potential $\Phi=\Phi_{\infty}$

$$
\begin{aligned}
\lambda \Delta \Phi & =n[\Phi]-p[\Phi]-C(x), \quad x \in \Omega \\
n[\Phi] & =g(\alpha[\Phi]+\Phi) \\
p[\Phi] & =g(\beta[\Phi]-\Phi)
\end{aligned}
$$

subject to homogeneous Neumann conditions

$$
\nabla \Phi \cdot \boldsymbol{\nu}=0 \quad \text { on } \partial \Omega
$$

The constants $\alpha[\Phi], \beta[\Phi]$ (so-called Fermi-levels), which determine the particle densities $n_{\infty}=n[\Phi], p_{\infty}=p[\Phi]$, are given by the normalisations

$$
\begin{aligned}
& \int_{\Omega} g(\alpha[\Phi]+\Phi) d x=N \\
& \int_{\Omega} g(\beta[\Phi]-\Phi) d x=P
\end{aligned}
$$

where (8) is assumed to hold for consistency reasons.

We refer to [GaGr89, CDMS99] for an analysis of (19)-(22) in the "linear" case $g(\sigma)=\exp (\sigma)$ (Boltzmann distribution) and to [BDM99, Unt97] for the model in "nonlinear" cases.

It turns out that (19)-(21) is a critical point of the functional

$$
\begin{aligned}
J_{\lambda}[\Phi]=\frac{\lambda}{2} \int_{\Omega}|\nabla \Phi|^{2} d x-\int_{\Omega} C(x) \Phi d x & \\
& +\int_{\Omega} G(\alpha[\Phi]+\Phi) d x+\int_{\Omega} G(\beta[\Phi]-\Phi) d x \\
& -N \alpha[\Phi]-P \beta[\Phi],
\end{aligned}
$$


where $G$ is a primitive of $g$. We shall show in the case $\bar{h}=\infty$ that $J_{\lambda}$ is a strictly convex, weakly lower semicontinuous, bounded from below functional on an apropriate convex set. The solution of (19)-(21) is therefore uniquely determined as the minimum of $J_{\lambda}$.

Actually, the constraints (23), (24) which determine $\alpha$ and $\beta$ makes the problem nonlocal and it turns out that a transformed functional is easier to handle (in case of $\bar{h}=\infty$ and for carrying out the limit $\lambda \rightarrow 0$, see Appendix A). This new functional reads

$$
E_{\lambda}(n, p)=\int_{\Omega} H(n) d x+\int_{\Omega} H(p) d x+\frac{1}{2 \lambda} \int_{\Omega}|\nabla V|^{2} d x
$$

where $\left(H^{\prime}\right)^{-1}=g_{\mid(0,+\infty)}$ (i.e. $\left.H^{\prime}=h\right)$ and where $V=V[n-p-C]$ is given by

$$
-\Delta V=n-p-C
$$

(with homogeneous Neuman boundary conditions) and has now to be minimized under the constraints

$$
N=\int_{\Omega} n(x) d x \quad \text { and } \quad P=\int_{\Omega} p(x) d x, \quad n \geq 0, p \geq 0 .
$$

The constants $\alpha$ and $\beta$ now simply appear as Lagrange multipliers associated to the normalization constraints for the $L^{1}$-norms of $n$ and $p$.

This allows us to state our first result.

Theorem. A. With the above notations, (19)-(21) has a unique solution.

The functional $E_{\lambda}$ is the one which is used to study in $L^{1}$ the asymptotic behaviour of drift-diffusion problems corresponding to a nonlinear diffusion (see [CJMTU00, BDM99] for the case with a Poisson coupling; see [AMTU98, AMT98, BiDo99] for the case with Poisson coupling and a linear diffusion). Such a framework is also especially convenient for the study of the so-called insulator limit (see [CDMS99] for a justification in terms of physical quantities) corresponding to $\lambda \rightarrow 0$, and as in [CDMS99] (this paper is basically a generalization of [CDMS99] emphasizing the abstract structure of the problem), we have to distinguish two regimes. 
Theorem. B. If either $N>\int_{\Omega} C^{+} d x$ or (equivalently) $P>\int_{\Omega} C^{-} d x$, then the solution of (19)-(21) converges as $\lambda \rightarrow 0$ to the solution of (19)-(21) with $\lambda=0$, which is unique. In the other case, there exist two measurable subsets of $\Omega, \Omega_{n}$ and $\Omega_{p}$, respectively, with $\Omega_{n} \subseteq\{C \geq 0\}, \Omega_{p} \subseteq\{C \leq 0\}$, and a unique solution $\left(n_{0}, p_{0}, V_{0}\right)$, which is the limit of $(n, p, V)$ as $\lambda \rightarrow 0$, such that

$$
\begin{aligned}
& -\Delta V_{0}=n_{0}-p_{0}-C, \quad \int_{\Omega} V_{0} d x=0, \quad \int_{\Omega} n_{0} d x=N, \quad \int_{\Omega} p_{0} d x=P, \\
& V_{0}=\operatorname{ess} \inf _{\Omega} V_{0} \quad \text { on } \quad \Omega_{n} \\
& \Delta V_{0}=C \quad \text { on } \Omega \backslash\left(\Omega_{n} \cup \Omega_{p}\right) \text {, } \\
& V_{0}=\operatorname{ess} \sup _{\Omega} V_{0} \text { on } \quad \Omega_{p}
\end{aligned}
$$

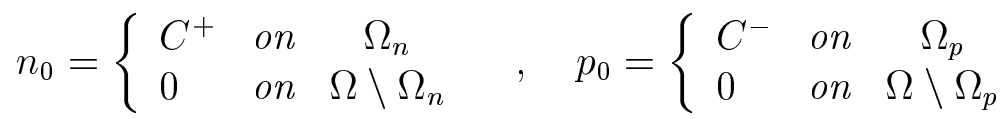

The conditions $N>\int_{\Omega} C^{+} d x$ and $P>\int_{\Omega} C^{-} d x$ are equivalent because of the global charge neutrality (25)). In the second case, $\Delta V_{0}=C$ on $\Omega \backslash$ $\left(\Omega_{n} \cup \Omega_{p}\right)$ and $v_{0}$ is constant on $\Omega_{n}$ and $\Omega_{p}$ where it reaches its maximum and its minimum respectively. Note that this double obstacle for $V$ is uniquely determined by the side condition $\int_{\Omega_{n}} C^{+} d x=N$.

The paper is organized as follows. The remainder of the first section is devoted to some preliminary results. A more detailed statement of Theorem A and its proof are given in Section 2, and Section 3 deals with the insulator limit. Remarks on the Legendre transform of the functional can be found in Appendix A and an extension to unbounded domains (corresponding to one species of particles, or two species when one looks for intermediate asymptotics) is given in Appendix B.

Before going further, let us fix some notations and state the detailed assumptions we shall use throughout this paper.

A.1 $\Omega \subset \mathbb{R}^{d}, d \in \mathbb{N}$, is a bounded, nonvoid domain.

A.2 $h: J \rightarrow \mathbb{R}, J=\mathbb{R}^{+}:=(0, \infty)$ or $J=\mathbb{R}_{0}^{+}:=[0, \infty)$, is continuous and strictly increasing.

$\underline{h}:=\inf _{J} h, \bar{h}:=\sup _{J} h . J=\mathbb{R}^{+}$iff $\underline{h}=-\infty$. 
A.3 $H: \mathbb{R}_{0}^{+} \rightarrow \mathbb{R}$ is continuous, differentiable on $\mathbb{R}^{+}$and

$$
\forall s \in \mathbb{R}^{+}: \quad H^{\prime}(s)=h(s) .
$$

A.4 $g:(-\infty, \bar{h}) \rightarrow J$ is the generalized inverse of $h$ as defined in (11).

A.5 $C \in L^{\infty}(\Omega) . \underline{C}=\operatorname{ess} \inf _{\Omega} C, \bar{C}=\operatorname{ess} \sup _{\Omega} C$.

Remark 1. a) $H$ is strictly convex.

b) $g$ is continuous and increasing, strictly increasing on $(\underline{h}, \bar{h})$ and $\lim _{t \rightarrow \bar{h}} g(t)=\infty$.

In the sequel we shall assume that assumptions A.1 and A.5 are always satisfied. and

Let $f \in L^{1}(\Omega)$ with $\int_{\Omega} f d x=0$. If there is $V \in H^{1}(\Omega)$ with $\int_{\Omega} V d x=0$

$$
\forall \phi \in H^{1}(\Omega): \quad \int_{\Omega} \nabla V \cdot \nabla \phi d x=\int_{\Omega} f \phi d x
$$

- which is, e.g., the case for all $f \in L^{1}(\Omega) \cap H^{-1}(\Omega)$ with $\int_{\Omega} f d x=0$ - then $V$ will be uniquely determined (by $f$ ) and we will set $V=V[f]$ and certainly

$$
\int_{\Omega}|\nabla V[f]|^{2} d x<\infty
$$

We observe: $V[f]$ is a weak solution of

$$
-\Delta V=f
$$

subject to homogeneous Neumann boundary conditions. However, if, for a given $f$, no such $V \in H^{1}(\Omega)$ exists, then we set

$$
\int_{\Omega}|\nabla V[f]|^{2} d x=\infty
$$

We introduce for $N, P \in \mathbb{R}^{+}$with $N-P=\int_{\Omega} C d x$ the set

$$
\mathcal{C}:=\left\{(n, p) \in L_{+}^{1}(\Omega) \times L_{+}^{1}(\Omega): \int_{\Omega} n d x=N, \int_{\Omega} p d x=P\right\},
$$

and we define for $\lambda \in \mathbb{R}^{+}$,

$$
\begin{aligned}
E_{\lambda}: \mathcal{C} \rightarrow \mathbb{R} \cup\{\infty\}, & \\
E_{\lambda}(n, p) & =\int_{\Omega} H(n) d x+\int_{\Omega} H(p) d x+\frac{1}{2 \lambda} \int_{\Omega}|\nabla V[n-p-C]|^{2} d x,
\end{aligned}
$$

where we make use of Jensen's inequality ensuring that $E_{\lambda}$ is bounded below. 


\subsection{A Semilinear Elliptic PDE of Order Two}

In this section we shall derive several auxiliary results.

Proposition 1. Assume A.1 and A.5 and let $\lambda \in \mathbb{R}^{+}$. Let $I \subseteq \mathbb{R}$ be a nonvoid, open interval and let $m: I \rightarrow \mathbb{R}$ be continuous and increasing with

$$
-\infty \leq \inf _{I} m<\underline{C} \leq 0, \quad 0 \leq \bar{C}<\sup _{I} m \leq \infty .
$$

Then there is exactly one $\Phi \in H^{1}(\Omega)$ with $\Phi(x) \in I$ for almost all $x \in \Omega$ and

$$
m(\Phi) \phi \in L^{1}(\Omega), \quad-\lambda \int_{\Omega} \nabla \Phi \cdot \nabla \phi=\int_{\Omega} m(\Phi) \phi d x-\int_{\Omega} C \phi d x,
$$

for all $\phi \in H^{1}(\Omega)$. Furthermore, $\Phi$ has the following properties:

1. $\Phi \in L^{\infty}(\Omega), \quad \underline{C} \leq m\left(\operatorname{ess} \inf _{\Omega} \Phi\right) \leq m\left(\operatorname{ess} \sup _{\Omega} \Phi\right) \leq \bar{C}$, in particular: there is a constant $\hat{\Phi} \in \mathbb{R}^{+}$such that

$$
\forall \lambda \in \mathbb{R}^{+}: \quad\|\Phi\|_{L^{\infty}(\Omega)} \leq \hat{\Phi}
$$

2. $\Phi$ is the unique weak solution (in $H^{1}(\Omega)$ ) of the semilinear elliptic PDE

$$
\lambda \Delta \Phi=m(\Phi)-C,
$$

subject to homogeneous Neumann boundary conditions.

3. $\int_{\Omega} m(\Phi) d x=\int_{\Omega} C d x$

Proof. Let

$$
M: I \rightarrow \mathbb{R}, \quad M(t):=\int_{t_{0}}^{t} m(s) d s,
$$

where $t_{0} \in I$ with $m\left(t_{0}\right)=0$. Since $m\left(t\left(<0\right.\right.$ for $t<t_{0}$ and since $m(t)>0$ for $t>t_{0}$, the function $M$ is a non-negative primitive of $m$. Furthermore, since $m$ is increasing, $M$ is convex. We introduce the functional

$$
\begin{aligned}
F_{\lambda}: H^{1}(\Omega) & \rightarrow \mathbb{R} \cup\{\infty\}, \\
F_{\lambda}(\eta) & =\left\{\begin{array}{cl}
\frac{\lambda}{2} \int_{\Omega}|\nabla \eta|^{2} d x+\int_{\Omega} M(\eta) d x-\int_{\Omega} C \eta d x & \text { if } \eta \in \mathcal{C}_{0}, \\
\infty & \text { if } \eta \notin \mathcal{C}_{0}
\end{array},\right.
\end{aligned}
$$


where

$$
\mathcal{C}_{0}=\left\{\eta \in H^{1}(\Omega): \eta(x) \in I \text { for almost all } x \in \Omega\right\}
$$

We certainly have $\inf _{\mathcal{C}_{0}} F_{\lambda} \in \mathbb{R}$. Let $\left(\eta_{k}\right)_{k \in \mathbb{N}}$ be a minimizing sequence of $F_{\lambda}$. By assumption we have for almost all $x \in \Omega$,

$$
\lim _{t \rightarrow \inf I}(m(t)-C(x))<0, \quad \lim _{t \rightarrow \sup I}(m(t)-C(x))>0,
$$

hence there is a compact interval $[a, b] \subseteq I$ (which is, by the way, independent of $\lambda$ ) such that $t \mapsto M(t)-C(x) t$ is decreasing on (inf $I, a$ ) and increasing on $(b, \sup I)$, i.e. for almost all $x \in \Omega$ :

$$
\begin{aligned}
& \forall t \in(\inf I, a): \quad M(t)-C(x) t \geq M(a)-C(x) a, \\
& \forall t \in(b, \sup I): \quad M(t)-C(x) t \geq M(b)-C(x) b .
\end{aligned}
$$

Now we set for $k \in \mathbb{N}$,

$$
\left[\eta_{k}\right]:=\min \left\{b, \max \left\{a, \eta_{k}\right\}\right\}
$$

Then $\left(\left[\eta_{k}\right]\right)_{k \in \mathbb{N}}$ is a sequence in $H^{1}(\Omega)$ because cutting maps $H^{1}(\Omega)$ (continuously) into itself. Furthermore, cutting does not increase the $L^{2}(\Omega)$-norms of the gradients [Zie89]. Hence

$$
\forall k \in \mathbb{N}: \quad F_{\lambda}\left(\left[\eta_{k}\right]\right) \leq F_{\lambda}\left(\eta_{k}\right),
$$

and therefore $\left(\left[\eta_{k}\right]\right)_{k \in \mathbb{N}}$ is a minimizing sequence of $F_{\lambda}$ as well. By passing to a subsequence (but without changing notation) we have

$$
\left[\eta_{k}\right] \rightarrow \Phi \in L^{\infty}(\Omega) \text { weak }^{*} \text { in } L^{\infty}(\Omega) \text { as } k \rightarrow \infty
$$

and therefore - since $L^{\infty}(\Omega) \subseteq L^{2}(\Omega)$ and since the $L^{2}\left(\Omega: \mathbb{R}^{d}\right)$-norms of $\left(\nabla\left[\eta_{k}\right]\right)_{k \in \mathbb{N}}$ are uniformly bounded - we have

$$
\left[\eta_{k}\right] \rightarrow \Phi \in H^{1}(\Omega) \text { weakly in } H^{1}(\Omega) \text { as } k \rightarrow \infty .
$$

We readily deduce from a lower semicontinuity argument that $\Phi$ is a minimizer of $F_{\lambda}$ in $H^{1}(\Omega)$. Since $F_{\lambda}$ is strictly convex due to the quadratic leading term, $\Phi$ is the unique minimizer of $F_{\lambda}$ in $H^{1}(\Omega)$. The uniform bounds 
$\eta_{\lambda}(x) \in[a, b] \subseteq I$ for almost all $x \in \Omega$ give $m(\Phi) \in L^{\infty}(\Omega)$ and the associated Euler-Lagrange equations

$$
\forall \phi \in H^{1}(\Omega):-\lambda \int_{\Omega} \nabla \Phi \cdot \nabla \phi=\int_{\Omega} m(\Phi) \phi d x-\int_{\Omega} C \phi d x
$$

easily follow. The proof of the remaining statements is left to the reader.

In particular cases the result of Proposition 1 can be extended as follows.

Proposition 2. Assume A.1 and A.5 and let $\lambda \in \mathbb{R}^{+}$. Let $I \subset \mathbb{R}$ be an open, nonvoid interval with inf $I \in \mathbb{R}$ or $\sup I \in \mathbb{R}$. Let $m: I \rightarrow \mathbb{R}$ be continuous and increasing. Assume furthermore one of the following conditions

1. If $\inf I \in \mathbb{R}$ and if

$$
\inf _{I} m=\underline{C} \leq 0, \quad 0 \leq \bar{C}<\sup _{I} m \leq \infty,
$$

then we set

$$
m_{0}:[\inf I, \sup I) \rightarrow[\underline{C}, \infty), \quad m_{0}(\rho)=\left\{\begin{array}{r}
\underline{C} \quad \text { if } \quad \inf _{I}=\rho \\
m(\rho) \quad \text { if } \quad \inf _{I}<\rho
\end{array}\right.
$$

2. If $\sup I \in \mathbb{R}$ and if

$$
-\infty \leq \inf _{I} m<\underline{C} \leq 0, \quad 0 \leq \bar{C}=\sup _{I} m
$$

then we e set

$$
m_{0}:(\inf I, \sup I] \rightarrow(-\infty, \bar{C}], \quad m_{0}(\rho)=\left\{\begin{array}{rll}
m(\rho) & \text { if } & \rho<\sup I \\
\bar{C} & \text { if } \quad \rho=\sup I
\end{array}\right.
$$

3. If $\inf I, \sup I \in \mathbb{R}$ and if

$$
\inf _{I} m=\underline{C} \leq 0, \quad 0 \leq \bar{C}=\sup _{I} m,
$$

then we set

$$
m_{0}:[\inf I, \sup I] \rightarrow[\underline{C}, \infty), \quad m_{0}(\rho)=\left\{\begin{array}{rll}
\underline{C} & \text { if } \rho=\inf _{I} \\
m(\rho) & \text { if } \quad \inf _{I}<\rho \\
\bar{C} & \text { if } \rho=\sup I
\end{array}\right.
$$


Then there is exactly one $\Phi \in H^{1}(\Omega)$ with $\Phi(x) \in \operatorname{dom}\left(m_{0}\right)$ for almost all $x \in \Omega$ and

$$
m_{0}(\Phi) \phi \in L^{1}(\Omega), \quad-\lambda \int_{\Omega} \nabla \Phi \cdot \nabla \phi=\int_{\Omega} m_{0}(\Phi) \phi d x-\int_{\Omega} C \phi d x,
$$

for all $\phi \in H^{1}(\Omega)$. Furthermore, $\Phi$ has the following properties:

1. $\Phi \in L^{\infty}(\Omega), \quad \underline{C} \leq m_{0}\left(\operatorname{ess} \inf _{\Omega} \Phi\right) \leq m_{0}\left(\operatorname{ess} \sup _{\Omega} \Phi\right) \leq \bar{C}$, in particular: there is a constant $\hat{\Phi} \in \mathbb{R}^{+}$such that

$$
\forall \lambda \in \mathbb{R}^{+}: \quad\|\Phi\|_{L^{\infty}(\Omega)} \leq \hat{\Phi}
$$

2. $\Phi$ is the unique weak solution (in $H^{1}(\Omega)$ ) of the semilinear elliptic PDE

$$
\lambda \Delta \Phi=m_{0}(\Phi)-C,
$$

subject to homogeneous Neumann boundary conditions.

3. $\int_{\Omega} m_{0}(\Phi) d x=\int_{\Omega} C d x$.

Proof. We consider case 1. Cases 2. and 3. can be treated in analogy. We define the function

$$
\begin{aligned}
& m_{1}:(-\infty, \sup I) \rightarrow \mathbb{R}, \\
& m_{1}(\rho)=\left\{\begin{aligned}
\underline{C}+(\rho-\inf I) & \text { if } \quad \rho \in(-\infty, \inf I] \\
m(\rho) & \text { if } \quad \rho \in(\inf I, \sup I)
\end{aligned}\right.
\end{aligned}
$$

Since $\inf m_{1}=-\infty<\underline{C}$, we can apply proposition 1 to obtain:

There is exactly one $\Phi_{1} \in H^{1}(\Omega)$ such that $\Phi_{1}(x) \in(-\infty, \sup I)$ for almost all $x \in \Omega$ and

$$
m_{1}\left(\Phi_{1}\right) \phi \in L^{1}(\Omega), \quad-\lambda \int_{\Omega} \nabla \Phi_{1} \cdot \nabla \phi=\int_{\Omega} m_{1}\left(\Phi_{1}\right) \phi d x-\int_{\Omega} C \phi d x,
$$

for all $\phi \in H^{1}(\Omega)$. Furthermore, $\Phi_{1}$ has the following properties:

$$
\text { 1. } \Phi_{1} \in L^{\infty}(\Omega), \quad \underline{C} \leq m_{1}\left(\operatorname{ess} \inf _{\Omega} \Phi_{1}\right) \leq m_{1}\left(\operatorname{ess} \sup _{\Omega} \Phi_{1}\right) \leq \bar{C},
$$


2. $\Phi_{1}$ is the unique weak solution (in $H^{1}(\Omega)$ ) of the semilinear elliptic PDE

$$
\lambda \Delta \Phi_{1}=m_{1}\left(\Phi_{1}\right)-C,
$$

subject to homogeneous Neumann boundary conditions.

3. $\int_{\Omega} m_{1}\left(\Phi_{1}\right) d x=\int_{\Omega} C d x$.

$m_{1}\left(\Phi_{1}\right) \phi \in L^{1}(\Omega), \quad-\lambda \int_{\Omega} \nabla \Phi_{1} \cdot \nabla \phi=\int_{\Omega} m_{1}\left(\Phi_{1}\right) \phi d x-\int_{\Omega} C \phi d x$,

We deduce from estimate 1.: $m_{1}\left(\Phi_{1}\right)=m_{0}\left(\Phi_{1}\right)$. Hence for all $\phi \in H^{1}(\Omega)$,

$$
m_{0}\left(\Phi_{1}\right) \phi \in L^{1}(\Omega), \quad-\lambda \int_{\Omega} \nabla \Phi_{1} \cdot \nabla \phi=\int_{\Omega} m_{0}\left(\Phi_{1}\right) \phi d x-\int_{\Omega} C \phi d x,
$$

$\Phi_{1}(x) \in \operatorname{dom}\left(m_{0}\right)=[\inf I, \sup I)$ for almost all $x \in \Omega$,

$$
\Phi_{1} \in L^{\infty}(\Omega), \quad \underline{C} \leq m_{0}\left(\operatorname{ess} \inf _{\Omega} \Phi_{1}\right) \leq m_{0}\left(\operatorname{ess}_{\Omega} \Phi_{1}\right) \leq \bar{C},
$$

$\Phi_{1}$ is a weak solution (in $H^{1}(\Omega)$ ) of the semilinear elliptic PDE

$$
\lambda \Delta \Phi_{1}=m_{0}\left(\Phi_{1}\right)-C,
$$

subject to homogeneous Neumann boundary conditions, and

$\int_{\Omega} m_{0}\left(\Phi_{1}\right) d x=\int_{\Omega} C d x$.

Now let $\Phi^{*}(x) \in \operatorname{dom}\left(m_{0}\right)$ for almost all $x \in \Omega$ and

$$
m_{0}\left(\Phi^{*}\right) \phi \in L^{1}(\Omega), \quad-\lambda \int_{\Omega} \nabla \Phi^{*} \cdot \nabla \phi=\int_{\Omega} m_{0}\left(\Phi^{*}\right) \phi d x-\int_{\Omega} C \phi d x .
$$

Then $m_{0}\left(\Phi^{*}\right)=m_{1}\left(\Phi^{*}\right)$ as well and we deduce $\Phi^{*}=\Phi_{1}$ from the uniqueness result cited above.

The verification that the semilinear elliptic PDE

$$
\lambda \Delta \Phi=m_{0}(\Phi)-C,
$$

subject to homogeneous Neumann boundary conditions and $\int_{\Omega} m_{0}(\Phi) d x=\int_{\Omega} C d x$, has a unique solution in $H^{1}(\Omega)$ is left to the reader. 


\subsection{An Abstract Variational Problem}

In this section we consider the limit $\lambda \rightarrow 0$ of an abstract minimization problems with a class of functionals containing $E_{\lambda}$.

Theorem 3. Let $(\mathfrak{B},\|\|$.$) be a Banach space and let$

$$
\mathcal{C} \subseteq \mathfrak{B}
$$

be nonvoid, convex and weakly closed in $\mathfrak{B}$. Let

$$
E, F: \mathcal{C} \rightarrow \mathbb{R} \cup\{\infty\} \text {, be bounded below with } \inf _{\mathcal{C}} E<\infty, \inf _{\mathcal{C}} F<\infty \text {. }
$$

We set

$$
\mathcal{C}^{*}:=\{x \in \mathcal{C}: E(x)<\infty\} .
$$

For $\lambda \in \mathbb{R}^{+}$let $x_{\lambda} \in \mathcal{C}$.

Assume

1. $x_{\lambda}$ is for each $\lambda \in \mathbb{R}^{+}$a minimizer of $E_{\lambda}:=E+\lambda^{-1} F$ in $\mathcal{C}$.

2. $x_{\lambda} \rightarrow x_{0}$ weakly in $\mathfrak{B}$ as $\lambda \rightarrow 0$.

Then

a) $\limsup _{\lambda \rightarrow 0} F\left(x_{\lambda}\right) \leq \inf _{\mathcal{C}^{*}} F$.

b) If $F$ is weakly lower sequentially continuous at $x_{0}$, then

$$
F\left(x_{0}\right) \leq \inf _{\mathcal{C}^{*}} F
$$

c) If $F$ is weakly lower sequentially continuous at $x_{0}$ and if $E\left(x_{0}\right)<\infty$, then $x_{0}$ is a minimizer of $F$ in $\mathcal{C}^{*}$, i.e.

$$
F\left(x_{0}\right)=\inf _{\mathcal{C}^{*}} F
$$

d) If $x^{*}$ is a minimizer of $F$ in $\mathcal{C}^{*}$, then

$$
\limsup _{\lambda \rightarrow 0} E\left(x_{\lambda}\right) \leq E\left(x^{*}\right)
$$


e) If $x^{*}$ is a minimizer of $F$ in $\mathcal{C}^{*}$ and if $E$ is weakly lower sequentially continuous at $x_{0}$, then

$$
E\left(x_{0}\right) \leq E\left(x^{*}\right)
$$

f) If $E$ and $F$ are weakly lower sequentially continuous at $x_{0}$ and if $E\left(x_{0}\right)<$ $\infty$, then $x_{0}$ is a minimizer of $F$ in $\mathcal{C}^{*}$ whose "energy" $E\left(x_{0}\right)$ is less or equal the energy $E\left(x^{*}\right)$ of any minimizer $x^{*}$ of $F$ in $\mathcal{C}^{*}$.

Proof. a) Let $-\rho \in \mathbb{R}$ be a lower bound of $E$ in $\mathcal{C}$. Let $x \in \mathcal{C}$ with $E(x)<\infty$. Since $\lambda>0$ we have

$$
\lambda\left(E\left(x_{\lambda}\right)+\rho\right)+F\left(x_{\lambda}\right) \leq \lambda(E(x)+\rho)+F(x) .
$$

Hence by non-negativity of $E\left(x_{\lambda}\right)+\rho$,

$$
\limsup _{\lambda \rightarrow 0} F\left(x_{\lambda}\right) \leq F(x) \text {. }
$$

b) follows from a).

c) If $E\left(x_{0}\right)<\infty$, then $x_{0}$ is by b) a minimizer of $F$ in $\mathcal{C}^{*}$.

d) Let $-\rho \in \mathbb{R}$ be a lower bound of $E, F$. Let $x_{*} \in \mathcal{C}^{*}$ be a minimizer of $F$ in $\mathcal{C}^{*}$. Then $E\left(x^{*}\right)<\infty$ and we obtain for all $\lambda \in \mathbb{R}^{+}$the estimate

$$
\left(E\left(x_{\lambda}\right)+\rho\right)+\frac{1}{\lambda}\left(F\left(x_{\lambda}\right)+\rho\right) \leq\left(E\left(x_{*}\right)+\rho\right)+\frac{1}{\lambda}\left(F\left(x_{*}\right)+\rho\right),
$$

from which we deduce $E\left(x_{\lambda}\right)<\infty$, hence $x_{\lambda} \in \mathcal{C}^{*}$, and due to $F\left(x_{*}\right) \leq F\left(x_{\lambda}\right)$,

$$
E\left(x_{\lambda}\right) \leq E\left(x_{*}\right) .
$$

e) follows from d) and f) follows from a)-e).

\section{The Main Results}

\section{$2.1 \lambda \in \mathbb{R}^{+}$}

The main result of this section is

Theorem 4. Assume A.1-A.5. Let $\lambda \in \mathbb{R}^{+}$and let $N, P \in \mathbb{R}^{+}$with

$$
N-P=\int_{\Omega} C d x
$$

Then: 
1. The functional $E_{\lambda}$ has a unique minimizer $\left(n_{\lambda}, p_{\lambda}\right)$ in $\mathcal{C}$.

2. $n_{\lambda}, p_{\lambda} \in L^{\infty}(\Omega)$ with

$$
n_{\lambda} \leq \bar{C}+\frac{P}{\operatorname{meas}(\Omega)}, \quad p_{\lambda} \leq-\underline{C}+\frac{N}{\operatorname{meas}(\Omega)} .
$$

3. There are $\alpha_{\lambda}, \beta_{\lambda} \in \mathbb{R}$ such that

$$
\begin{aligned}
& n_{\lambda}=g\left(\alpha_{\lambda}-\lambda^{-1} V_{\lambda}\right), \\
& p_{\lambda}=g\left(\beta_{\lambda}+\lambda^{-1} V_{\lambda}\right),
\end{aligned}
$$

where $V_{\lambda}=V\left[n_{\lambda}-p_{\lambda}-C\right]$, hence if one sets $\Phi_{\lambda}:=-\lambda^{-1} V_{\lambda}$, then

$$
\lambda \Delta \Phi_{\lambda}=g\left(\alpha_{\lambda}+\Phi_{\lambda}\right)-g\left(\beta_{\lambda}-\Phi_{\lambda}\right)-C, \quad \int_{\Omega} \Phi_{\lambda} d x=0,
$$

subject to homogeneous Neumann boundary conditions and

$$
\int_{\Omega} g\left(\alpha_{\lambda}+\Phi_{\lambda}\right) d x=N, \quad \int_{\Omega} g\left(\beta_{\lambda}-\Phi_{\lambda}\right) d x=P .
$$

Proof. Due to the lack of coercitivity (in case of $\bar{h}<\infty$ ) of $E_{\lambda}$ it is not obvious that $E_{\lambda}$ has a minimizer in $\mathcal{C}$. The argumentation will be based settled on the semilinear equation

$$
\lambda \Delta \Psi=g(\alpha+\Psi)-g(\beta-\Psi)-C
$$

subject to homogeneous Neumann boundary conditions. In (26) the constants $\alpha, \beta$ are a priori unknown. Our program is to prove: There are $\alpha, \beta \in \mathbb{R}$ such that $\int_{\Omega} g(\alpha+\Psi) d x=N, \int_{\Omega} g(\beta-\Psi) d x=P$.

We consider for $c \in(-\infty, 2 \bar{h})$ the semilinear elliptic PDE

$$
\lambda \Delta \Phi=g(c+\Phi)-g(-\Phi)-C
$$

subject to homogeneous Neumann boundary conditions. This problem fits with

$$
m(.)=g(c+.)-g(-.), \quad I=(-\bar{h}, \bar{h}-c)
$$


to Proposition 1: there is a unique $\Phi_{c} \in H^{1}(\Omega)$ which solves (27) subject to homogeneous Neumann boundary conditions. Furthermore, $\Phi_{c}$ belongs to $L^{\infty}(\Omega)$ and if we set

$$
\begin{gathered}
n_{c}=g\left(c+\Phi_{c}\right), \quad p_{c}=g\left(-\Phi_{c}\right), \\
\overline{\Phi_{c}}:=\operatorname{ess} \sup _{\Omega} \Phi_{c}, \quad \underline{\Phi_{c}}:=\operatorname{ess} \inf _{\Omega} \Phi_{c}
\end{gathered}
$$

then we deduce from (27) the estimates

$$
g\left(c+\overline{\Phi_{c}}\right)-g\left(-\overline{\Phi_{c}}\right) \leq \bar{C}, \quad g\left(c+\underline{\Phi_{c}}\right)-g\left(-\underline{\Phi_{c}}\right) \geq \underline{C} .
$$

Hence the functions $n_{c}, p_{c}$ belong to $L^{\infty}(\Omega)$. Let us introduce

$$
N_{c}=\int_{\Omega} n_{c} d x=\int_{\Omega} g\left(c+\Phi_{c}\right) d x, \quad P_{c}=\int_{\Omega} p_{c} d x=\int_{\Omega} g\left(-\Phi_{c}\right) d x
$$

Then $N_{c}-P_{c}=\int_{\Omega} C d x$ and we have

$$
\left\|n_{c}\right\|_{L^{\infty}(\Omega)} \leq \bar{C}+\frac{P_{c}}{\operatorname{meas}(\Omega)}, \quad\left\|p_{c}\right\|_{L^{\infty}(\Omega)} \leq-\underline{C}+\frac{N_{c}}{\operatorname{meas}(\Omega)} .
$$

We have to prove: there is $c \in \mathbb{R}$ such that $N_{c}=N, P_{c}=P$. This is shown in several steps.

$c \mapsto\left(N_{c}, P_{c}\right)$ is continuous. Indeed, let $c \in(-\infty, 2 \bar{h})$ and let $\left(c_{k}\right)_{k \in \mathbb{N}}$ be a sequence in $(-\infty, 2 \bar{h})$ with $\lim _{k \rightarrow \infty} c_{k}=c$ and let $\left(c_{k_{1}(k)}\right)_{k \in \mathbb{N}}$ be a subsequence of $\left(c_{k}\right)_{k \in \mathbb{N}}$. We have to prove: there is a subsequence $\left(c_{k_{2}(k)}\right)_{k \in \mathbb{N}}$ of $\left(c_{k_{1}(k)}\right)_{k \in \mathbb{N}}$ with

$$
\lim _{k \rightarrow \infty} N_{c_{k_{2}(k)}}=N_{c}, \quad \lim _{k \rightarrow \infty} P_{c_{k_{2}(k)}}=P_{c}
$$

We deduce from (28): There are $K_{0}, K_{1} \in \mathbb{N}$ such that for all $k \in \mathbb{N}$

$$
\left\|n_{c_{k}}\right\|_{L^{\infty}(\Omega)},\left\|p_{c_{k}}\right\|_{L^{\infty}(\Omega)} \leq K_{1}, \quad k \geq K_{0} .
$$

Hence there is a subsequence $\left(k_{3}(k)\right)_{k \in \mathbb{N}}$ of $\left(k_{1}(k)\right)_{k \in \mathbb{N}}$ and there are $n_{0}, p_{0} \in$ $L^{\infty}(\Omega)$ with

$$
n_{k_{3}(k)} \rightarrow n_{0}, p_{k_{3}(k)} \rightarrow n_{0} \quad \text { weak }^{*} \text { in } L^{\infty}(\Omega) \text { as } k \rightarrow \infty
$$


We deduce:

$$
\Phi_{k_{3}(k)} \rightarrow \Phi_{0} \quad \text { strongly in } H^{1}(\Omega) \text { as } k \rightarrow \infty
$$

where

$$
\lambda \Delta \Phi_{0}=n_{0}-p_{0}-C,
$$

subject to homogeneous Neumann boundary conditions. Hence there is a subsequence $\left(k_{2}(k)\right)_{k \in \mathbb{N}}$ of $\left(k_{3}(k)\right)_{k \in \mathbb{N}}$ with

$$
\Phi_{k_{2}(k)}(x) \rightarrow \Phi_{0}(x) \quad \text { for almost all } x \in \Omega \text { as } k \rightarrow \infty \text {. }
$$

We deduce

$$
\begin{aligned}
& n_{k_{2}(k)}(x) \\
= & g\left(c_{k_{2}(k)}+\Phi_{k_{2}(k)}\right)(x) \rightarrow g\left(c+\Phi_{0}\right)(x) \text { for almost all } x \in \Omega \text { with } k \rightarrow \infty,
\end{aligned}
$$

$p_{k_{2}(k)}(x)=g\left(-\Phi_{k_{2}(k)}\right)(x) \rightarrow g\left(-\Phi_{0}\right)(x) \quad$ for almost all $x \in \Omega$ with $k \rightarrow \infty$.

Hence $n_{0}=n_{c}, \Phi_{0}=\Phi_{c}, p_{0}=p_{c}$ and therefore due to weak ${ }^{*}$ convergence in $L^{\infty}(\Omega), \lim _{k \rightarrow \infty} N_{c_{k_{2}(k)}}=N_{c}, \lim _{k \rightarrow \infty} P_{c_{k_{2}(k)}}=P_{c}$.

$\lim _{c \rightarrow 2 \bar{h}} N_{c}=\infty, \lim _{c \rightarrow 2 \bar{h}} P_{c}=\infty$.

Here we only consider $\bar{h}<\infty$. The case $\bar{h}=\infty$ can be treated similiarily. Due to $N_{c}=P_{c}+\int_{\Omega} C d x$ it sufficies to prove: $\lim _{c \rightarrow \infty} P_{c}=\infty$. According to $c+\Phi_{c}(x)<\bar{h}$ and $-\Phi_{c}(x)<\bar{h}$ for almost all $x \in \Omega$, we deduce

$$
c-\bar{h}<-\Phi_{c}(x)<\bar{h} \quad \text { for almost all } x \in \Omega \text {. }
$$

Hence

$$
\lim _{c \rightarrow 2 \bar{h}} P_{c}=\lim _{c \rightarrow 2 \bar{h}} \int_{\Omega} g\left(-\Phi_{c}\right) d x \geq \lim _{c \rightarrow 2 \bar{h}} g(c-\bar{h}) \operatorname{meas}(\Omega)=\infty .
$$

If $c<c_{1}<2 \bar{h}$, then $c+\Phi_{c} \leq c_{1}+\Phi_{c_{1}}$ and $\Phi_{c} \geq \Phi_{c_{1}}$. Indeed we have

$$
\lambda \Delta \Phi_{c}=g\left(c+\Phi_{c}\right)-g\left(-\Phi_{c}\right)-C, \quad \lambda \Delta \Phi_{c_{1}}=g\left(c_{1}+\Phi_{c_{1}}\right)-g\left(-\Phi_{c_{1}}\right)-C .
$$


Substraction and testing with $\left[\left(c+\Phi_{c}\right)-\left(c_{1}+\Phi_{c_{1}}\right)\right]^{+} \in H^{1}(\Omega)$ gives

$$
\begin{aligned}
& -\lambda \int_{\Omega}\left|\nabla\left[\left(c+\Phi_{c}\right)-\left(c_{1}+\Phi_{c_{1}}\right)\right]^{+}\right|^{2} d x \\
= & \int_{\Omega}\left(g\left(c+\Phi_{c}\right)-g\left(-\Phi_{c}\right)-\left(g\left(c_{1}+\Phi_{c_{1}}\right)-g\left(-\Phi_{c_{1}}\right)\right)\left[c+\Phi_{c}\right)-\left(c_{1}+\Phi_{c_{1}}\right)\right]^{+} d x \geq 0,
\end{aligned}
$$

because $g$ is increasing and $c<c_{1}$ implies $-\Phi_{c}(x) \leq-\Phi_{c_{1}}(x)$ for all $x \in \Omega$ with $c+\Phi_{c}(x) \geq c_{1}+\Phi_{c_{1}}(x)$. Hence $c+\Phi_{c}(x) \leq c_{1}+\Phi_{c_{1}}(x)$ for almost all $x \in \Omega$. The estimate $\Phi_{c}(x) \geq \Phi_{c_{1}}(x)$ for almost all $x \in \Omega$ follows from a similiar argument by using the test function $\left[\Phi_{c_{1}}-\Phi_{c}\right]^{+} \in H^{1}(\Omega)$.

In particular we deduce

$$
\text { If } c<c_{1}<2 \bar{h} \text {, then } N_{c} \leq N_{c_{1}}, P_{c} \leq P_{c_{1}}
$$

Behaviour of $n_{c}, p_{c}$ as $c \rightarrow-\infty$ Let $c_{\circ} \in(-\infty, 2 \bar{h})$ be fixed. Then due to previous estimates we have for all $c \in\left(-\infty, c_{\circ}\right]$,

$$
\left\|n_{c}\right\|_{L^{\infty}(\Omega)} \leq \bar{C}+\frac{P_{c}}{\operatorname{meas}(\Omega)} \leq\|C\|_{L^{\infty}(\Omega)}+\frac{N_{c_{\circ}}+P_{c_{\circ}}}{\operatorname{meas}(\Omega)}=K_{\circ},
$$

where $K_{\circ} \in \mathbb{R}^{+}$is independent of $c \in\left(-\infty, c_{\circ}\right]$. In analogy we obtain

$$
\left\|p_{c}\right\|_{L^{\infty}(\Omega)} \leq K_{\circ}
$$

We deduce: there is a sequence $\left(c_{k}\right)_{k \in \mathbb{N}} \in\left(-\infty, c_{\circ}\right]$ with $\lim _{k \rightarrow \infty} c_{k}=-\infty$ and there are $n_{-}, p_{-} \in L^{\infty}(\Omega)$ with

$$
n_{c_{k}} \rightarrow n_{-}, p_{c_{k}} \rightarrow p_{-} \text {weak }^{*} \text { in } L^{\infty}(\Omega) \text { as } k \rightarrow \infty,
$$

and certainly

$$
\lim _{k \rightarrow \infty} N_{c_{k}}=N_{-}, \quad \lim _{k \rightarrow \infty} P_{c_{k}}=P_{-} .
$$

We also have for all $k \in \mathbb{N}$,

$$
\lambda \Delta \Phi_{c_{k}}=n_{c_{k}}-p_{c_{k}}-C,
$$

such that

$$
\left\|\nabla \Phi_{c_{k}}\right\|_{L^{2}\left(\Omega: \mathbb{R}^{d}\right)} \leq K_{1}
$$


for some $K_{1} \in \mathbb{R}^{+}$independent of $k \in \mathbb{N}$. Hence - possibly after passing to a subsequence but without changing notations -

$$
\Phi_{c_{k}}-\frac{1}{\operatorname{meas}(\Omega)} \int_{\Omega} \Phi_{c_{k}} d x \rightarrow V_{-} \in H^{1}(\Omega) \quad \text { strongly in } H^{1}(\Omega) \text { as } k \rightarrow \infty
$$

where $\int_{\Omega} V_{-} d x=0$. We introduce

$$
\omega:=\lim _{k \rightarrow \infty} \int_{\Omega} \Phi_{c_{k}} d x \in \mathbb{R} \cup\{\infty\}
$$

where we made use of the fact that $c \mapsto \int_{\Omega} \Phi_{c} d x$ is increasing. Now it is easy to see: Either $\omega=\infty$ with

$$
\Phi_{c_{k}} \rightarrow \infty \text { almost everywhere on } \Omega \text { as } k \rightarrow \infty
$$

or

$$
\Phi_{c_{k}} \rightarrow \Phi_{-} \quad \text { strongly in } H^{1}(\Omega) \text { and almost everywhere on } \Omega \text { as } k \rightarrow \infty \text {, }
$$

where

$$
\lambda \Delta \Phi_{-}=n_{-}-p_{-}-C
$$

subject to homogeneous Neumann boundary conditions. We also introduce

$$
\omega^{*}:=\lim _{k \rightarrow \infty} \int_{\Omega}\left(c_{k}+\Phi_{c_{k}}\right) d x \in \mathbb{R} \cup\{-\infty\},
$$

where we made use of the fact that $c \mapsto \int_{\Omega}\left(c+\Phi_{c}\right) d x$ is decreasing. We deduce as above: Either $\omega^{*}=-\infty$ with

$$
c_{k}+\Phi_{c_{k}} \rightarrow-\infty \quad \text { almost everywhere on } \Omega \text { as } k \rightarrow \infty
$$

or

$c_{k}+\Phi_{c_{k}} \rightarrow \Phi_{*} \quad$ strongly in $H^{1}(\Omega)$ and almost everywhere on $\Omega$ as $k \rightarrow \infty$, where

$$
\lambda \Delta \Phi_{*}=n_{-}-p_{-}-C,
$$


subject to homogeneous Neumann boundary conditions.

Existence of $c \in(-\infty, 2 \bar{h})$ with $N_{c}=N, P_{c}=P$. Due to the previous results it remains to prove: $\lim _{k \rightarrow \infty} N_{c_{k}}=0$ or $\lim _{k \rightarrow \infty} P_{c_{k}}=0$. We distinguish with respect to $\int_{\Omega} C d x$.

$\int_{\Omega} C d x>0$ : In that case $\omega \in \mathbb{R}$ is not possible, because

$$
\begin{aligned}
\liminf _{k \rightarrow \infty} \int_{\Omega} n_{c_{k}} d x=\liminf _{k \rightarrow \infty} \int_{\Omega} g\left(c_{k}\right. & \left.+\Phi_{c_{k}}\right) d x \\
=0 & =-\liminf _{k \rightarrow \infty} \int_{\Omega} p_{c_{k}} d x-\int_{\Omega} C d x<0
\end{aligned}
$$

would follow. Hence $\omega=\infty$ and therefore by Lebesgue's dominated convergence Theorem,

$$
\lim _{k \rightarrow-\infty} P_{c_{k}}=\int_{\Omega} g\left(-\Phi_{c_{k}}\right) d x=0
$$

$\int_{\Omega} C d x=0$ : It is easy to see: If $\omega=\infty$, then $\lim _{k \rightarrow \infty} P_{c_{k}}=0$, or if $\omega \in \mathbb{R}$, then $\lim _{k \rightarrow \infty} N_{c_{k}}=0$.

$\int_{\Omega} C d x<0$ : We consider the value of $\omega^{*}$, proceed as in case of $\int_{\Omega} C d x>0$ and conclude $\lim _{k \rightarrow \infty} N_{c_{k}}=0$.

End of proof. We have shown: there is $c \in \mathbb{R}$ such that

$$
\lambda \Delta \Phi_{c}=g\left(c+\Phi_{c}\right)-g\left(-\Phi_{c}\right)-C
$$

subject to homogeneous Neumann boundary conditions has a solution $\Phi_{c} \in$ $H^{1}(\Omega)$ with $\int_{\Omega} g\left(c+\Phi_{c}\right) d x=N, \int_{\Omega} g\left(-\Phi_{c}\right) d x=P$. We set

$$
\begin{gathered}
V_{\lambda}:=-\lambda \Phi_{c}+\frac{\lambda}{\operatorname{meas}(\Omega} \int_{\Omega} \Phi_{c} d x, \\
\alpha_{\lambda}=c-\frac{1}{\operatorname{meas}(\Omega)} \int_{\Omega} \Phi_{c} d x, \quad \beta_{\lambda}=\frac{1}{\operatorname{meas}(\Omega)} \int_{\Omega} \Phi_{c} d x,
\end{gathered}
$$

and

$$
n_{\lambda}=n_{c}=g\left(\alpha_{\lambda}-\lambda^{-1} V_{\lambda}\right), \quad p_{\lambda}=p_{c}=g\left(\beta_{\lambda}+\lambda^{-1} V_{\lambda}\right)
$$


Then it is easy to see that $\left(n_{\lambda}, p_{\lambda}\right) \in \mathcal{C}$ satisfy the variational inequalities

$$
\begin{array}{lll}
h\left(n_{\lambda}\right)+\lambda^{-1} V_{\lambda}=\alpha_{\lambda} \quad \text { on } & \left\{n_{\lambda}>0\right\} \\
h\left(n_{\lambda}\right)+\lambda^{-1} V_{\lambda} \geq \alpha_{\lambda} \quad \text { on } & \left\{n_{\lambda}=0\right\} \\
h\left(p_{\lambda}\right)-\lambda^{-1} V_{\lambda}=\beta_{\lambda} \quad \text { on } \quad\left\{p_{\lambda}>0\right\} \\
h\left(p_{\lambda}\right)-\lambda^{-1} V_{\lambda} \geq \beta_{\lambda} \quad \text { on } \quad\left\{p_{\lambda}=0\right\}
\end{array},
$$

where $V_{\lambda}=V\left[n_{\lambda}-p_{\lambda}-C\right]$. It is left to the reader to verify that the validity of these variational inequalities ensures that $\left(n_{\lambda}, p_{\lambda}\right)$ is the unique minimizer of $E_{\lambda}$ in $\mathcal{C}$ and to prove that $n_{\lambda}, p_{\lambda}$ have the properties as specified in the Theorem.

\section{$2.2 \lambda \rightarrow 0$}

The main result of this section is

Theorem 5. Assume A.1-A.5. Let $N, P \in \mathbb{R}^{+}$with

$$
N-P=\int_{\Omega} C d x
$$

For $\lambda \in \mathbb{R}^{+}$let $\left(n_{\lambda}, p_{\lambda}\right)$ be the unique minimizer of $E_{\lambda}$ in $\mathcal{C}$, see theorem 4 , and $V_{\lambda}=V\left[n_{\lambda}-p_{\lambda}-C\right]$. Then:

1. There is $\left(n_{0}, p_{0}\right) \in \mathcal{C} \cap\left(L^{\infty}(\Omega)\right)^{2}$ such that

$$
n_{\lambda} \rightarrow n_{0}, p_{\lambda} \rightarrow p_{0} \quad \text { weak }{ }^{*} \text { in } L^{\infty}(\Omega) \text { as } \lambda \rightarrow 0,
$$

2. $V_{\lambda} \rightarrow V_{0}=V\left[n_{0}-p_{0}-C\right]$ strongly in $H^{1}(\Omega)$ as $\lambda \rightarrow 0$.

Furthermore,

3. If $N>\int_{\Omega} C^{+} d x, P>\int_{\Omega} C^{-} d x$, then $V_{0}=0$ and $\left(n_{0}, p_{0}\right)$ is the unique minimizer of

$$
E: \mathcal{C} \rightarrow \mathbb{R} \cup\{\infty\}, \quad E(n, p)=\int_{\Omega} H(n) d x+\int_{\Omega} H(p) d x
$$


in

$$
\mathcal{C}^{0}=\{(n, p) \in \mathcal{C}: n-p=C\} \neq \emptyset
$$

i.e. there is a unique $\gamma \in \mathbb{R}$ and a unique $\rho \in L^{\infty}(\Omega)$ with

$$
\begin{array}{cl}
\max \left\{-C^{+},-C^{-}\right\} \leq \rho, \quad \int_{\Omega} \rho d x=N-\int_{\Omega} C^{+} d x=P-\int_{\Omega} C^{-} d x, \\
h\left(C^{+}+\rho\right)+h\left(C^{-}+\rho\right)=\gamma \quad \text { on } & \left\{\rho>-C^{+}\right\} \cap\left\{\rho>-C^{-}\right\} \\
h\left(C^{+}+\rho\right)+h\left(C^{-}+\rho\right) \geq \gamma & \text { on } \quad\left\{\rho=-C^{+}\right\} \cup\left\{\rho=-C^{-}\right\}
\end{array}
$$

determining $n_{0}, p_{0}$ via

$$
n_{0}=C^{+}+\rho, \quad p_{0}=C^{-}+\rho .
$$

4. If $N=\int_{\Omega} C^{+} d x, P=\int_{\Omega} C^{-} d x$, then $V_{0}=0$ and

$$
n_{0}=C^{+}, \quad p_{0}=C^{-} .
$$

5. If $N<\int_{\Omega} C^{+} d x, P<\int_{\Omega} C^{-} d x$ then there is a unique pair $\left(\Omega_{n}, \Omega_{p}\right)$ of measurable subsets of $\Omega$ such that

$$
\begin{gathered}
\operatorname{meas}\left(\Omega_{n} \cap \Omega_{p}\right)=0, \quad \Omega_{n} \subset\{C \geq 0\}, \Omega_{p} \subset\{C \leq 0\}, \\
\int_{\Omega_{n}} C^{+} d x=N, \quad \int_{\Omega_{p}} C^{-} d x=P, \\
n_{0}=C^{+} \quad \text { on } \quad \Omega_{n}, \quad p_{0}=C^{-} \text {on } \quad \Omega_{p} \\
n_{0}=0 \quad p_{0}=0 \quad \text { on } \Omega \backslash \Omega_{p}
\end{gathered}
$$


Proof. Let $\left(\lambda_{k}\right)_{k \in \mathbb{N}}$ be a sequence in $\mathbb{R}^{+}$with $\lim _{k \rightarrow \infty} \lambda_{k}=0$. By the uniform estimates on $\left\|n_{\lambda}\right\|_{L^{\infty}(\Omega)},\left\|p_{\lambda}\right\|_{L^{\infty}(\Omega)}$ there is a subsequence $\left(\lambda_{k_{1}(k)}\right)_{k \in \mathbb{N}}$ of $\left(\lambda_{k}\right)_{k \in \mathbb{N}}$ and there is $\left(n_{0}, p_{0}\right) \in \mathcal{C}$ such that

$$
n_{\lambda_{k_{1}(k)}} \rightarrow n_{0}, p_{\lambda_{k_{1}(k)}} \rightarrow p_{0} \quad \text { weak* in } L^{\infty}(\Omega) \text { as } k \rightarrow \infty .
$$

Hence

$$
V_{\lambda_{k_{1}(k)}} \rightarrow V_{0}=V\left[n_{0}-p_{0}-C\right] \text { strongly in } H^{1}(\Omega) \text { as } k \rightarrow \infty,
$$

hence after passing to a subsequence $\left(k_{2}(k)\right)_{k \in \mathbb{N}}$ of $\left(k_{1}(k)\right)_{k \in \mathbb{N}}$,

$$
V_{\lambda_{k_{2}(k)}} \rightarrow V_{0} \text { for almost all } x \in \Omega \text { as } k \rightarrow \infty \text {. }
$$

We have to prove that $\left(n_{0}, p_{0}\right)$ are actually independent of the sequence $\left(\lambda_{k}\right)_{k \in \mathbb{N}}$. We proceed by a case-distinction and set

$$
F: \mathcal{C} \rightarrow \mathbb{R}_{0}^{+} \cup\{\infty\}, \quad F(n, p)=\int_{\Omega}|\nabla V[n-p-C]|^{2} d x .
$$

The main ingredient is the application of theorem 3 .

Case I: $N>\int_{\Omega} C^{+}$and $P>\int_{\Omega} C^{-}$.

We have $\mathcal{C}^{0} \neq \emptyset$. By theorem 3 , the pair $\left(n_{0}, p_{0}\right)$ minimizes $E$ in the set of all minimizers of $F$ for which $E$ is bounded, i.e. in $\left\{(n, p) \in \mathcal{C}^{0}: E(n, p)<\infty\right\}$. By strict convexity, the functional $E$ has exactly one minimizer in that set. It is left to the reader to deduce the variational inequalities as specified in 3 .

Case II: $N=\int_{\Omega} C^{+} d x$ and $P=\int_{\Omega} C^{-} d x$.

With the notations of Case I we have $\mathcal{C}^{0}=\left\{\left(C^{+}, C^{-}\right)\right\}$. The statement follows from theorem 3 .

Case III: $N<\int_{\Omega} C^{+} d x$ and $P<\int_{\Omega} C^{-} d x$. In this case the set $\mathcal{C}^{0}$ is void. However, we deduce from theorem 3 that $\left(n_{0}, p_{0}\right)$ - we note that $E\left(n_{0}, p_{0}\right)<\infty$ because $n_{0}, p_{0} \in L^{\infty}(\Omega)$ - is a minimizer of $F$ in $\mathcal{C}^{*}=\{(n, p) \in$ $\mathcal{C}: E(n, p)<\infty\}$.

We observe: $V_{0}$ does not vanish identically. (Otherwise $n_{0}-p_{0}=C$ implying $\left.N \geq \int_{\Omega} C^{+} d x, P \geq \int_{\Omega} C^{-} d x\right)$. Hence due to $\int_{\Omega} V_{0} d x=0$,

$$
0<\operatorname{meas}\left(\left\{V_{0}<0\right\}\right), \operatorname{meas}\left(\left\{V_{0}>0\right\}\right)<\operatorname{meas}(\Omega),
$$

and therefore

$$
\operatorname{ess} \inf _{\Omega} V_{0}<\operatorname{ess} \sup _{\Omega} V_{0}
$$


The sequence $\left(\alpha_{\lambda_{k_{2}(k)}}-\lambda_{k_{2}(k)}^{-1} V_{\lambda_{k_{2}(k)}}(x)\right)_{k \in \mathbb{N}}$ is, for almost all $x \in\left\{V_{0}<0\right\}$, bounded above as $k \rightarrow \infty$. Hence $\lim _{k \rightarrow \infty} \alpha_{\lambda_{k_{2}(k)}}=-\infty$. By the wellknown identification of weak ${ }^{*}$ limits in $L^{\infty}$ and pointwise almost-everywhere limits of sequences of functions we obtain for almost all $x \in \Omega$ : If $\left(\alpha_{\lambda_{k_{2}(k)}}-\right.$ $\left.\lambda_{k_{2}(k)}^{-1} V_{\lambda_{k_{2}(k)}}(x)\right)_{k \in \mathbb{N}}$ converges in $\mathbb{R} \cup\{ \pm \infty\}$, then $n_{0}(x)=\lim _{k \rightarrow \infty} g\left(\alpha_{\lambda_{k_{2}(k)}}-\right.$ $\left.\lambda_{k_{2}(k)}^{-1} V_{\lambda_{k_{2}(k)}}(x)\right)$. Hence, up to a set of measure zero,

$$
\left\{V_{0}>0\right\} \subseteq\left\{n_{0}=0\right\} .
$$

Now take $x \in \Omega$ such that $\lim _{k \rightarrow \infty} V_{\lambda_{k_{2}(k)}}(x)=V_{0}(x)$, that the sequence $\left(\alpha_{k_{2}(k)}-\lambda_{k_{2}(k)}^{-1} V_{\lambda_{k_{2}(k)}}(x)\right)_{k \in \mathbb{N}}$ is bounded above and $V_{0}(x)>\operatorname{ess~inf}_{\Omega} V_{0}$. Then there is $z \in \Omega$ with $\lim _{k \rightarrow \infty} V_{\lambda_{k_{2}(k)}}(z)=V_{0}(z)=V_{0}(x)-\eta$ with some $\eta \in \mathbb{R}^{+}$ and the sequence $\left(\alpha_{k_{2}(k)}-\lambda_{k_{2}(k)}^{-1} V_{\lambda_{k_{2}(k)}}(z)\right)_{k \in \mathbb{N}}$ is bounded above, too. Since $\lim _{k \rightarrow \infty}\left(V_{\lambda_{k_{2}(k)}}(x)-V_{\lambda_{k_{2}(k)}}(z)\right) / \lambda_{k_{2}(k)}=\infty$, we obtain up to a set of measure zero:

If $\lim _{k \rightarrow \infty} V_{\lambda_{k_{2}(k)}}(x)>\operatorname{essinf}_{\Omega} V_{0}$, then

$$
\lim _{k \rightarrow \infty}\left(\alpha_{k_{2}(k)}-\lambda_{k_{2}(k)}^{-1} V_{\lambda_{k_{2}(k)}}(x)\right)=-\infty \text {, and } n_{0}(x)=0 \text { follows. }
$$

Hence

$$
\Omega_{n}=\left\{n_{0}>0\right\} \subseteq\left\{V_{0}=\operatorname{ess} \inf _{\Omega} V_{0}\right\}
$$

and in analogy

$$
\left\{V_{0}<0\right\} \subseteq\left\{p_{0}=0\right\}, \quad \Omega_{p}=\left\{p_{0}>0\right\} \subseteq\left\{V_{0}=\underset{\Omega}{\left.\operatorname{ess} \sup _{0} V_{0}\right\}},\right.
$$

in particular $\left\{n_{0}>0\right\} \cap\left\{p_{0}>0\right\}=\emptyset$. Now it is easy to see that the triple $\left(n_{0}, p_{0}, V_{0}\right)$ has the properties as specified in 5 . of the theorem. It remains to be shown that there is at most one minimizer of $F$ in $\mathcal{C}^{*}$. Assume that $\left(n_{*}, p_{*}\right)$ is a minimizer of $F$ in $\mathcal{C}^{*}$. If $n_{0}-p_{0} \neq n_{*}-p_{*}$, then by strict convexity we would obtain

$$
\begin{aligned}
F\left(\left(n_{0}+n_{*}\right) / 2,\left(p_{0}+p_{*}\right) / 2\right) & \\
& =\int_{\Omega}\left|\nabla V\left[\left(\left(n_{0}-p_{0}-C\right) / 2\right)+\left(\left(n_{*}-p_{*}-C\right) / 2\right)\right]\right|^{2} d x \\
<\frac{1}{2} \int_{\Omega}\left|\nabla V\left[n_{0}-p_{0}-C\right]\right|^{2} d x+\frac{1}{2} \int_{\Omega}\left|\nabla V\left[n_{*}-p_{*}-C\right]\right|^{2} d x & =\frac{F\left(n_{0}, p_{0}\right)+F\left(n_{*}, p_{*}\right)}{2} .
\end{aligned}
$$


Hence $n_{*}-p_{*}=n_{0}-p_{0}$ and therefore $n_{*}=n_{0}+\rho, p_{*}=p_{0}+\rho$ for some $\rho \in L^{1}(\Omega)$ with $\int_{\Omega} \rho d x=0$. We have on $\Omega \backslash \Omega_{n} n_{0}=0$, hence $\rho \geq 0$, and we have in analogy $\rho \geq 0$ on $\Omega \backslash \Omega_{p}$. Since $\Omega_{n}$ and $\Omega_{p}$ are disjoint we obtain $\rho \geq 0$, hence $\rho=0$.

Remark 2. a) Point 3 of Theorem 5 represents the case, where the limit satisfies local charge-neutrality everywhere in $\Omega$.

b) The limit $V_{0}$ in point 5 of Theorem 5 is the unique solution of a double obstacle problem. The coincidence set $\Omega_{n}$ of the smaller obstacle $\operatorname{ess}_{\inf } V_{0}$ is the set where the electron density $n$ equals the doping profile, the coincidence set $\Omega_{p}$ of the larger obstacle $\operatorname{ess}^{\sup _{\Omega}} V_{0}$ is the set where the hole density $p$ equals the negative doping profile and the noncoincidence set $\Omega \backslash\left(\Omega_{n} \cup \Omega_{p}\right)$ is the depletion (vacuum) region where $n=p=0$. Note that the double obstacle problem for $V_{0}$ is somewhat nonstandard since the obstacles are not a-priorily given but determined by the constraints $\int_{\Omega_{n}} C^{+} d x=N$ and $\int_{\Omega_{p}} C^{-} d x=P$ on the coincidence sets. However, the a-posteriori regularity theory for the

free boundaries is by now standard [Caf00]. They are (locally) $C^{1, \alpha}$-surfaces if the inhomogeneity $C(x)$ is $C^{\alpha}$.

\section{Appendix A: Analysis of $J_{\lambda}$}

We shall explain in a simple case the connection between the functionals $J_{\lambda}$ and $E_{\lambda}$, thus introducing for simplicity several technical assumptions that can be removed with a more detailed analysis.

Consider as in the introduction the functional

$$
\begin{aligned}
J_{\lambda}[\Phi]=\frac{\lambda}{2} \int_{\Omega}|\nabla \Phi|^{2} d x-\int_{\Omega} C(x) \Phi d x & \\
+\int_{\Omega} G(\alpha[\Phi]+\Phi) d x+\int_{\Omega} G(\beta[\Phi]-\Phi) d x & -N \alpha[\Phi]-P \beta[\Phi],
\end{aligned}
$$

where $\alpha=\alpha[\Phi]$ and $\beta=\beta[\Phi]$ are determined by the condition

$$
\begin{aligned}
& \int_{\Omega} g(\alpha[\Phi]+\Phi) d x=N \\
& \int_{\Omega} g(\beta[\Phi]-\Phi) d x=P
\end{aligned}
$$


with $g=G^{\prime} \geq 0, g \not \equiv 0, \lim _{s \rightarrow-\infty} g(s)=0$ and bounded $\Omega \subseteq \mathbb{R}^{d}$. Note that for $\Phi \in L^{\infty}(\Omega)$, the map $\alpha \mapsto \int_{\Omega} g(\alpha+\Phi) d x$ is well defined, continuous by Lebesgue's theorem of dominated convergence and converges to 0 and $+\infty$ as $\alpha$ tends to $-\infty$ and $+\infty$, respectively. Analogous properties of course hold for the map $\beta \mapsto \int_{\Omega} g(\beta-\Phi) d x$. Note also that if $g$ is nondecreasing (which is the case if $G$ is convex), then $g^{\prime}$ is a positive measure on $\mathbb{R}$. If we further assume that $g^{\prime} \in L_{\text {loc }}^{\infty}(\mathbb{R})$ and $g^{\prime}>0$ on $\mathbb{R}$, then the functionals $\alpha$ [.] and $\beta[$. are actually $C^{1}$ on $L^{\infty}(\Omega)$, and

$$
\begin{aligned}
& d \alpha[\phi] \cdot \chi=-\frac{\int_{\Omega} g^{\prime}(\alpha[\phi]+\phi) \chi d x}{\int_{\Omega} g^{\prime}(\alpha[\phi]+\phi) d x} \\
& d \beta[\phi] \cdot \chi=\frac{\int_{\Omega} g^{\prime}(\beta[\phi]-\phi) \chi d x}{\int_{\Omega} g^{\prime}(\beta[\phi]-\phi) d x}
\end{aligned}
$$

Proposition 6. Assume A1 and A5. If $G \in C^{2}(\mathbb{R}), G^{\prime \prime}=g^{\prime} \in L_{\text {loc }}^{\infty}(\mathbb{R})$, $g^{\prime}>0$ on $\mathbb{R}$ and $\lim _{s \rightarrow+\infty} g(s)=+\infty, \lim _{s \rightarrow-\infty} g(s)=0$, then $\Phi_{\lambda}$ is a weak solution of

$$
\lambda \Delta \Phi_{\lambda}=g\left(\alpha\left[\Phi_{\lambda}\right]+\Phi_{\lambda}\right)-g\left(\beta\left[\Phi_{\lambda}\right]-\Phi_{\lambda}\right)-C(x)
$$

in $H^{1} \cap L^{\infty}(\Omega)$ subject to homogeneous Neumann boundary conditions if and only if it is a critical point of $J_{\lambda}[\Phi]$ in $H^{1} \cap L^{\infty}(\Omega)$. The functional $J_{\lambda}[\Phi]$ is strictly convex, so $\Phi_{\lambda}$ is unique.

Proof. A straightforward calculation shows that for any $\phi, \chi \in H^{1} \cap L^{\infty}(\Omega)$,

$$
\begin{array}{r}
d J_{\lambda}[\phi] \cdot \chi=\int_{\Omega}[\lambda \nabla \phi \cdot \nabla \chi+(g(\alpha[\phi]+\phi)-g(\beta[\phi]-\phi)-C(x)) \chi] d x \\
+\left[\int_{\Omega}(g(\alpha[\phi]+\phi) d x-N] d \alpha[\phi] \cdot \chi\right. \\
+\left[\int_{\Omega}(g(\beta[\phi]-\phi) d x-P] d \beta[\phi] \cdot \chi .\right.
\end{array}
$$

Using the constraints (29) and (30), we find that $d J_{\lambda}\left[\Phi_{\lambda}\right] \cdot \chi=0$ exactly means that $\Phi_{\lambda}$ is a weak solution of (31).

To prove the convexity of $J_{\lambda}$, we shall consider $\phi_{1}$ and $\phi_{2}$ in $H^{1} \cap L^{\infty}(\Omega)$. Denoting $\psi=\phi_{2}-\phi_{1}, \phi^{t}=t \phi_{1}+(1-t) \phi_{2}$ and $j(t)=J_{\lambda}\left[\phi^{t}\right]$ for any $t \in(0,1)$, 
a direct computation shows that

$$
j^{\prime}(t)=\int_{\Omega}\left[\lambda \nabla \phi^{t} \cdot \nabla \psi+\left(g\left(\alpha\left[\phi^{t}\right]+\phi^{t}\right)+g\left(\beta\left[\phi^{t}\right]-\phi^{t}\right)-C(x)\right) \psi\right] d x,
$$

and

$$
\begin{array}{r}
j^{\prime \prime}(t)=\lambda \int_{\Omega}|\nabla \psi|^{2} d x+\int_{\Omega}\left[g^{\prime}\left(\alpha\left[\phi^{t}\right]+\phi^{t}\right)-g^{\prime}\left(\beta\left[\phi^{t}\right]-\phi^{t}\right)\right]|\psi|^{2} d x \\
-\frac{\left(\int_{\Omega} g^{\prime}\left(\alpha\left[\phi^{t}\right]+\phi^{t}\right) \psi d x\right)^{2}}{\int_{\Omega} g^{\prime}\left(\alpha\left[\phi^{t}\right]+\phi^{t}\right) d x}-\frac{\left(\int_{\Omega} g^{\prime}\left(\beta\left[\phi^{t}\right]-\phi^{t}\right) \psi d x\right)^{2}}{\int_{\Omega} g^{\prime}\left(\beta\left[\phi^{t}\right]-\phi^{t}\right) d x} \\
\geq \lambda \int_{\Omega}|\nabla \psi|^{2} d x
\end{array}
$$

by the Cauchy-Schwarz inequality.

To prove the boundedness from below of $J_{\lambda}$, it is actually simpler to consider the functional

$$
E_{\lambda}(n, p)=\int_{\Omega} H(n) d x+\int_{\Omega} H(p) d x+\frac{1}{2 \lambda} \int_{\Omega}|\nabla V[n-p-C]|^{2} d x
$$

with $H^{\prime}=h$ and $g$ related according to A4. Note that A2 and A3 are consequences of the assumptions of Proposition 6. This functional has (according to Theorem 3) a unique minimizer $\left(n_{\lambda}, p_{\lambda}\right)$ in $\mathcal{C}$ such that

$$
\begin{gathered}
\Phi_{\lambda}=\lambda^{-1} V_{\lambda} \\
\lambda \Delta \Phi_{\lambda}=n_{\lambda}-p_{\lambda}-C \\
\text { with } n_{\lambda}=g\left(\alpha\left[\Phi_{\lambda}\right]+\Phi_{\lambda}\right) \text { and } p_{\lambda}=g\left(\beta\left[\Phi_{\lambda}\right]-\Phi_{\lambda}\right),
\end{gathered}
$$

which is a critical point of $J_{\lambda}$, so (by convexity) $J_{\lambda}$ is bounded from below by $J_{\lambda}\left[\Phi_{\lambda}\right]$.

Note also that the constraints (29) and (30) can be rewritten as

$$
\begin{gathered}
\alpha\left[\Phi_{\lambda}\right]+\Phi_{\lambda}=h\left(n_{\lambda}\right) \\
\beta\left[\Phi_{\lambda}\right]-\Phi_{\lambda}=h\left(p_{\lambda}\right)
\end{gathered}
$$

where we make use of $n_{\lambda}, p_{\lambda}>0$ on $\Omega$ due to $g>0$ on $\mathbb{R}$. This is why

$$
\begin{aligned}
\alpha\left[\Phi_{\lambda}\right] N & =\int_{\Omega}\left(h\left(n_{\lambda}\right)-\Phi_{\lambda}\right) n_{\lambda} d x \\
\beta\left[\Phi_{\lambda}\right] P & =\int_{\Omega}\left(h\left(p_{\lambda}\right)+\Phi_{\lambda}\right) p_{\lambda} d x
\end{aligned}
$$


and therefore

$$
\begin{aligned}
& J_{\lambda}\left[\Phi_{\lambda}\right]=-\frac{\lambda}{2} \int_{\Omega} \Phi_{\lambda} \Delta \Phi_{\lambda} d x+\int_{\Omega} G\left(\alpha\left[\Phi_{\lambda}\right]+\Phi_{\lambda}\right) d x+\int_{\Omega} G\left(\beta\left[\Phi_{\lambda}\right]-\Phi_{\lambda}\right) d x \\
& \quad-\int_{\Omega} C \Phi_{\lambda} d x-N \alpha\left[\Phi_{\lambda}\right]-P \beta\left[\Phi_{\lambda}\right] \\
&=-\frac{1}{2} \int_{\Omega}\left(n_{\lambda}-p_{\lambda}-C\right) \Phi_{\lambda} d x+\int_{\Omega}\left(G o h\left(n_{\lambda}\right)-n_{\lambda} h\left(n_{\lambda}\right)\right) d x \\
& \quad+\int_{\Omega}\left(G o h\left(p_{\lambda}\right)-p_{\lambda} h\left(p_{\lambda}\right)\right) d x+\int_{\Omega} \Phi_{\lambda}\left(n_{\lambda}-p_{\lambda}-C\right) d x \\
&=-\int_{\Omega} H\left(n_{\lambda}\right) d x-\int_{\Omega} H\left(p_{\lambda}\right) d x-\frac{1}{2 \lambda} \int_{\Omega}\left|\nabla V_{\lambda}\left[n_{\lambda}-p_{\lambda}-C\right]\right|^{2} d x \\
&=-E_{\lambda}\left[n_{\lambda}, p_{\lambda}\right]
\end{aligned}
$$

using the fact that $\frac{d}{d t}[t h(t)-G o h(t)]=h(t)$, and $H(t)=t h(t)-G o h(t)$ (up to a constant which is chosen here equal to 0$)$.

\section{Appendix B: Unbounded domains}

In this section we shall investigate (19)-(24) on unbounded domains. In this case the analysis has to overcome several additional difficulties and further assumptions are needed.

We put

$$
C_{c}^{\infty}(\bar{\Omega})=\left\{\phi \mid \Omega: \phi \in C_{c}^{\infty}\left(\mathbb{R}^{d}\right)\right\}
$$

and we assume

V.1 There is a linear operator $V: L_{l o c}^{1}(\bar{\Omega}) \supset \operatorname{dom}(V) \rightarrow L_{l o c}^{1}(\bar{\Omega})$, such that for all $f \in \operatorname{dom}(V)$, the function $V[f]$ is a weak solution of

$$
-\Delta V[f]=f, \quad+\text { homogeneous Neumann boundary conditions, }
$$

i.e.

$$
-\int_{\Omega} V[f] \Delta \phi d x=\int_{\Omega} f \phi d x, \quad \forall \phi \in C_{c}^{\infty}(\bar{\Omega})
$$


V.2 The set $\operatorname{dom}_{2}(V):=\left\{f \in \operatorname{dom}(V): \nabla V[f] \in L^{2}\left(\Omega: \mathbb{R}^{d}\right)\right\}$ contains

$$
\mathrm{T}:=\left\{\rho \in L^{\infty}(\Omega):(\operatorname{supp}(\rho) \subset \subset \bar{\Omega}) \wedge\left(\int_{\Omega} \rho d x=0\right)\right\},
$$

and for all $f \in \operatorname{dom}_{2}(V)$,

$$
\int_{\Omega} \nabla V[f] \cdot \nabla V[\rho] d x=\int_{\Omega} V[f] \rho d x, \quad \forall \rho \in \mathrm{T} .
$$

V.3 If $\left(f_{n}\right)_{n \in \mathbb{N}}$ is a sequence in $\operatorname{dom}_{2}(V)$ with

$$
f_{n} \rightarrow f, \quad \text { weakly in } L^{1}(\Omega) \text { as } n \rightarrow \infty,
$$

and if $\left(\nabla V\left[f_{n}\right]\right)_{n \in \mathbb{N}}$ is bounded in $L^{2}\left(\Omega: \mathbb{R}^{d}\right)$, then $f \in \operatorname{dom}(V)$ and

$$
\lim _{n \rightarrow \infty} \int_{\Omega} V\left[f_{n}\right] \phi d x=\int_{\Omega} V[f] \phi d x, \quad \forall \phi \in C_{c}^{\infty}(\bar{\Omega}) .
$$

Remark 3. a) It is easy to see that V.1, V.2, V.3 hold for bounded domains $\Omega$ where $\operatorname{dom}(V)$ is the set of all $f \in L^{1}(\Omega)$ with $\int_{\Omega} f d x=0$ for which a function $Z \in H^{1}(\Omega)$ exists such that $\int_{\Omega} Z d x=0$ and $\int \nabla Z \cdot \nabla \phi d x=$ $\int_{\Omega} f \phi d x$ for all $\phi \in H^{1}(\Omega)$, compare the definition of " $V[f]$ " in the previous sections.

b) Assumptions V.1-V.3 apply in particular to $\Omega=\mathbb{R}^{d}, d \in \mathbb{N}$, and to respective half-space problems, see the discussion below.

c) The verification of V.1-V.3 of the examples of b) rely on the knowledge of a Green's function. Whenever such a function is available, then one may proceed similiar as in b) to investigate the validity of V.1, V.2, V.3.

d) We note that (as e.g. in case of $\Omega=\mathbb{R}^{d}, d \geq 3$ ) the domain $\operatorname{dom}(V)$ of the operator $V[$.$] may consist of functions which do not satisfy the global$ electroneutrality condition $\int_{\Omega} f d x=0$.

Example $1 \Omega=\mathbb{R}$. We set $\operatorname{dom}(V)=L^{1}(\mathbb{R})$ and introduce for $x \in \mathbb{R}$ and for $f \in \operatorname{dom}(V)$,

$$
E(f)(x):=-\int_{-\infty}^{x} f(s) d s, \quad V(x):=\left\{\begin{array}{ll}
\int_{0}^{x} E(f)(s) d s \quad, \quad x \geq 0 \\
-\int_{x}^{0} E(f)(s) d s, & x<0
\end{array} .\right.
$$


It is easy to see: $E: \operatorname{dom}(V) \rightarrow L^{\infty}(\mathbb{R})$ is non-expansive, i.e. Lipschitzcontinuous with Lipschitz constant 1.

Furthermore, $V^{\prime}=E(f)$ and $V^{\prime \prime}=-f$ in the sense of distributions (see, e.g., [Rud]).

Hence $V: \operatorname{dom}(V) \rightarrow C^{1}(\mathbb{R})$ satisfies $\mathbf{V} \mathbf{1}$.

If $\rho \in L^{\infty}(\mathbb{R})$ has compact support and satisfies $\int_{\mathbb{R}} \rho d x=0$, then $E(\rho)$ has compact support, too. Since $E(\rho) \in L^{\infty}(\mathbb{R})$ we deduce $V^{\prime}[\rho]=E(\rho) \in L^{2}(\mathbb{R})$, thus $\mathrm{T}=\left\{\rho \in L^{\infty}(\mathbb{R}):(\operatorname{supp}(\rho) \subset \subset \mathbb{R}) \wedge\left(\int_{\mathbb{R}} \rho d x=0\right)\right\} \subseteq \operatorname{dom}_{2}(V)$.

Now let $f \in \operatorname{dom}_{2}(V)$ and let $\rho \in \mathrm{T}$. We take a sequence $\left(f_{n}\right)_{n \in \mathbb{N}}$ in $C_{c}^{\infty}(\mathbb{R})$ with $f_{n} \rightarrow f$ strongly in $L^{1}(\Omega)$ as $n \rightarrow \infty$ (see, e.g. [Ada]) and $\int_{\mathbb{R}} f_{n} d x=0$ for all $n \in \mathbb{N}$. Then $V\left[f_{n}\right] \in C_{c}^{\infty}(\mathbb{R})$ for all $n \in \mathbb{N}$ and therefore

$$
\int_{\mathbb{R}} \rho V\left[f_{n}\right] d x=-\int_{\mathbb{R}} V[\rho] V^{\prime \prime}\left[f_{n}\right], \quad n \in \mathbb{N} .
$$

Since $V[\rho]$ and $V^{\prime \prime}\left[f_{n}\right]$ are continuously differentiable and since $V[\rho]$ is compactly supported, we calculate by means of an integration by parts,

$$
-\int_{\mathbb{R}} V[\rho] V^{\prime \prime}\left[f_{n}\right]=\int_{\mathbb{R}} V^{\prime}\left[f_{n}\right] V^{\prime}[\rho] d x=\int_{\mathbb{R}} E\left(f_{n}\right) E(\rho) d x, \quad n \in \mathbb{N} .
$$

Since $E(\rho) \in L^{\infty}(\mathbb{R})$ is compactly supported and since $E\left(f_{n}\right) \rightarrow E(f)$ strongly in $L^{\infty}(\mathbb{R})$, we deduce $\int_{\mathbb{R}} E\left(f_{n}\right) E(\rho) d x \rightarrow \int_{\mathbb{R}} E(f) E(\rho) d x$ as $n \rightarrow$ $\infty$. On the other hand it is easy to see that $E\left(f_{n}\right) \rightarrow E(f)$ strongly in $L^{\infty}(\mathbb{R})$ implies $V\left[f_{n}\right] \rightarrow V[f]$ in $L_{l o c}^{1}(\mathbb{R})$ as $n \rightarrow \infty$. This settles $\int_{\mathbb{R}} \rho V\left[f_{n}\right] d x \rightarrow$ $\int_{\mathbb{R}} \rho V[f] d x$ as $n \rightarrow \infty$ and therefore

$$
\begin{aligned}
& \int_{\mathbb{R}} V^{\prime}[f] V^{\prime}[\rho] d x \\
& =\int_{\mathbb{R}} E(f) E(\rho) d x=\lim _{n \rightarrow \infty} \int_{\mathbb{R}} E\left(f_{n}\right) E(\rho) d x=\lim _{n \rightarrow \infty} \int_{\mathbb{R}} V\left[f_{n}\right] \rho d x \\
& \quad=\int_{\mathbb{R}} V[f] \rho d x
\end{aligned}
$$

It remains to verify $\mathbf{V . 3}$. If $f_{n} \rightarrow f$ weakly in $L^{1}(\mathbb{R})$, then $\left(f_{n}\right)_{n \in \mathbb{N}}$ is bounded in $L^{1}(\mathbb{R})$. Hence $\left(V^{\prime}\left[f_{n}\right]\right)_{n \in \mathbb{N}}=\left(E\left(f_{n}\right)\right)_{n \in \mathbb{N}}$ is bounded in $L^{\infty}(\mathbb{R})$. Furthermore, since the indicator function of $(-\infty, x)$ is in $L^{\infty}(\mathbb{R})$ for each $x \in \mathbb{R}$, we deduce $E\left(f_{n}\right)(x) \rightarrow E(f)(x)$ as $n \rightarrow \infty$ for all $x \in \mathbb{R}$. As a consequence, $V\left[f_{n}\right](x) \rightarrow V[f](x)$ for all $x \in \mathbb{R}$. Due to uniform boundedness 
of $\left(E\left(f_{n}\right)\right)_{n \in \mathbb{N}}$ in $L^{\infty}(\mathbb{R})$ we also have: $V\left[f_{n}\right] \rightarrow V[f]$ in $L_{\text {loc }}^{1}(\mathbb{R})$ as $n \rightarrow \infty$. Hence $V\left[f_{n}\right] \rightarrow V[f]$ in the sense of distributions as well.

Example $2 \Omega=\mathbb{R}^{2}$. We set $\operatorname{dom}(V):=L^{1}\left(\mathbb{R}^{2}\right)$. The Green's function of the Laplace operator is

$$
K(x):=-\frac{1}{2 \pi} \log |x|, \quad x \in \mathbb{R}^{2}, x \neq 0 .
$$

Since $K(x) \in L_{\text {loc }}^{1}\left(\mathbb{R}^{2}\right)$ we have for each $f \in L^{1}\left(\mathbb{R}^{2}\right)$,

$$
K \star f:=\int_{\mathbb{R}^{2}} K(.-y) f(y) d y \in L_{l o c}^{1}\left(\mathbb{R}^{2}\right) .
$$

For $f \in L^{1}\left(\mathbb{R}^{2}\right)$ we set

$$
V[f]:=-K \star f .
$$

$V[$.$] certainly satisfies \mathbf{V . 1}$.

We observe for $i=1,2$,

$$
\partial_{i} V[f]=-\left(\partial_{i} K\right) \star f, \quad \partial_{i} K(x)=-\frac{1}{2 \pi} \frac{1}{|x|} \frac{x_{i}}{|x|},
$$

in the sense of distributions.

In the sequel we shall use several estimates on $V[f]$ and on $\nabla V[f]$. These results can be found in [ArNi],

Proposition 7. (Follows from Lemma 3.3c) in [ArNi]) There is a positive real number $K_{8}$ such that: If

$$
f \in L^{1}\left(\mathbb{R}^{2}\right) \cap L^{2}\left(\mathbb{R}^{2}\right), \quad \text { and } \quad \int_{\mathbb{R}^{2}} f d x=0, \quad \text { and } \quad \int_{\mathbb{R}^{2}}|x||f(x)| d x<\infty,
$$

then $\nabla V[f] \in L^{2}\left(\mathbb{R}^{2}: \mathbb{R}^{2}\right)$ and

$$
\|\nabla V[f]\|_{L^{2}\left(\mathbb{R}^{2}: \mathbb{R}^{2}\right)} \leq K_{8}\left(\|f\|_{L^{2}\left(\mathbb{R}^{2}\right)}+\int_{\mathbb{R}^{2}}|x||f(x)| d x\right) .
$$

Proposition 8. (Follows from Lemma 3.1 in [ArNi]) There is a positive real number $K_{9}$ such that: If

$$
f \in L^{1}\left(\mathbb{R}^{2}\right), \quad \text { and } \quad \int_{\mathbb{R}^{2}} f d x=0
$$


then for all $x \in \mathbb{R}^{2}$,

$$
|V[f](x)| \leq K_{9}\left(\int_{\mathbb{R}^{2}}(1+|y|)|f(y)| d y+\left(\int_{\mathbb{R}^{2}}(1+|y|)^{2}|f(y)|^{2} d y\right)^{\frac{1}{2}}\right) .
$$

Proposition 9. (Follows from Lemma 3.1 in [ArNi]) There is a positive real number $K_{10}$ such that: If

$$
f \in L^{1}\left(\mathbb{R}^{2}\right), \quad \text { and } \quad \int_{\mathbb{R}^{2}} f d x=0,
$$

then $V[f] \in L^{6}\left(\mathbb{R}^{2}\right)$ with

$$
\|V[f]\|_{L^{6}\left(\mathbb{R}^{2}\right)} \leq K_{10} \cdot\left(\int_{\mathbb{R}^{2}}(1+|y|)|f(y)| d y+\left(\int_{\mathbb{R}^{2}}(1+|y|)^{\frac{3}{2}}|f(y)|^{\frac{3}{2}} d y\right)^{\frac{2}{3}}\right) .
$$

Now we shall verify $\mathbf{V} .2$. If $\rho \in L^{\infty}\left(\mathbb{R}^{2}\right)$ is compactly supported with $\int_{\mathbb{R}^{2}} \rho d x=0$, then $\rho$ fullfills the requirements of Proposition 7 . Hence $\nabla V[\rho] \in L^{2}\left(\mathbb{R}^{2}: \mathbb{R}^{2}\right)$ and therefore $\rho \in \operatorname{dom}_{2}(V)$.

Let $f \in \operatorname{dom}_{2}(V)$. Then the function $(x, y) \rightarrow K(x-y) f(y) \rho(x)$ belongs to $L^{1}\left(\mathbb{R}^{2} \times \mathbb{R}^{2}\right)$. Hence due to the Fubini-Tonelli Theorem,

$$
\begin{gathered}
\int_{\mathbb{R}^{2}} V[f] \rho d x=-\int_{\mathbb{R}^{2}}\left(\int_{\mathbb{R}^{d}} K(x-y) f(y) d y\right) \rho(x) d x \\
=-\int_{\mathbb{R}^{2} \times \mathbb{R}^{2}} K(x-y) f(y) \rho(x) d(x, y)=-\int_{\mathbb{R}^{2}}\left(\int_{\mathbb{R}^{2}} K(x-y) \rho(x) d x\right) f(y) d y \\
=-\int_{\mathbb{R}^{2}}\left(\int_{\mathbb{R}^{2}} K(y-x) \rho(x) d x\right) f(y) d y=\int_{\mathbb{R}^{2}} V[\rho] f d x,
\end{gathered}
$$

where we made use of $K(-x)=K(x)$. Thus, in order to verify $\mathbf{V} . \mathbf{2}$, it remains to prove

$$
\int_{\mathbb{R}^{2}} \nabla V[f] \cdot \nabla V[\rho] d x=\int_{\mathbb{R}^{2}} V[\rho] f d x
$$

We take a sequence $\left(\rho_{n}\right)_{n \in \mathbb{N}}$ in $C_{c}^{\infty}\left(\mathbb{R}^{2}\right)$ with $\lim _{n \rightarrow \infty}\left\|\rho-\rho_{n}\right\|_{L^{2}\left(\mathbb{R}^{2}\right)}=0$, see [Ada]. We can assume: There is $R \in(0, \infty)$ such that $\operatorname{supp}(\rho) \subseteq\{|y| \leq R\}$ and $\operatorname{supp}\left(\rho_{n}\right) \subseteq\{|y| \leq R\}$ for all $n \in \mathbb{N}$. We deduce from Proposition 7:

$$
\nabla V\left[\rho_{n}\right] \rightarrow \nabla V[\rho] \quad \text { strongly in } L^{2}\left(\mathbb{R}^{2}: \mathbb{R}^{2}\right) \text { as } n \rightarrow \infty .
$$


Furthermore, since $K \in L_{l o c}^{1}\left(\mathbb{R}^{2}\right)$ and since $\rho_{n} \in C_{c}^{\infty}\left(\mathbb{R}^{2}\right)$, we have $V\left[\rho_{n}\right] \in$ $C^{\infty}\left(\mathbb{R}^{2}\right)$ for all $n \in \mathbb{N}$, see e.g. [Ada].

Let us take $\Theta \in C^{\infty}(\mathbb{R})$ with $\Theta=1$ on $(-\infty, 0]$ and $\Theta=0$ on $[1, \infty)$. For $k \in \mathbb{N}$ we introduce

$$
\Theta_{k}: \mathbb{R}^{2} \rightarrow \mathbb{R}, \quad \Theta_{k}(x)=\Theta\left(\frac{|x|}{k}-1\right)
$$

Then $\Theta_{k} \in C_{c}^{\infty}\left(\mathbb{R}^{2}\right)$ for all $k \in \mathbb{N}$, hence $\Theta_{k} V\left[\rho_{n}\right] \in C_{c}^{\infty}\left(\mathbb{R}^{2}\right)$ for all $n, k \in \mathbb{N}$. We calculate for all $n, k \in \mathbb{N}$,

$$
\begin{gathered}
\int_{\mathbb{R}^{2}} f\left(\Theta_{k} V\left[\rho_{n}\right]\right) d x=-\int_{\mathbb{R}^{2}} V[f] \Delta\left(\Theta_{k} V\left[\rho_{n}\right]\right) d x=\int_{\mathbb{R}^{2}} \nabla V[f] \cdot \nabla\left(\Theta_{k} V\left[\rho_{n}\right]\right) d x \\
=\int_{\mathbb{R}^{2}}\left(\nabla V[f] \cdot \nabla \Theta_{k}\right) V\left[\rho_{n}\right] d x+\int_{\mathbb{R}^{2}}\left(\nabla V[f] \cdot \nabla V\left[\rho_{n}\right]\right) \Theta_{k} d x,
\end{gathered}
$$

where made use of $V\left[\rho_{n}\right] \in L^{\infty}\left(\mathbb{R}^{2}\right)$ (which follows from Proposition 8) and $\nabla V\left[\rho_{n}\right] \in L^{2}\left(\mathbb{R}^{2}: \mathbb{R}^{2}\right)$ for all $n \in \mathbb{N}$.

We consider the limit $n \rightarrow \infty$ now.

Due to Proposition 8 the sequence $\left(V\left[\rho_{n}\right]\right)_{n \in \mathbb{N}}$ is bounded in $L^{\infty}\left(\mathbb{R}^{2}\right)$. By Proposition 9 the sequence $\left(V\left[\rho_{n}\right]\right)_{n \in \mathbb{N}}$ converges strongly in $L^{6}\left(\mathbb{R}^{2}\right)$, to $V[\rho]$ as $n \rightarrow \infty$. Hence for all $k \in \mathbb{N}$,

$$
\begin{aligned}
\lim _{n \rightarrow \infty} \int_{\mathbb{R}^{2}} f\left(\Theta_{k} V\left[\rho_{n}\right]\right) d x & =\lim _{n \rightarrow \infty} \int_{\{k \leq|x| \leq 2 k\}} f\left(\Theta_{k} V\left[\rho_{n}\right]\right) d x \\
& =\int_{\{k \leq|x| \leq 2 k\}} f\left(\Theta_{k} V[\rho]\right) d x=\int_{\mathbb{R}^{d}} f\left(\Theta_{k} V[\rho]\right) d x
\end{aligned}
$$

Similiar argumentations (in particular exploiting the fact that each integration is in fact an integration over a fixed (i.e. independent of $n \in \mathbb{N}$ ) bounded domain) yield for all $k \in \mathbb{N}$,

$$
\begin{aligned}
& \lim _{n \rightarrow \infty} \int_{\mathbb{R}^{2}}\left(\nabla V[f] \cdot \nabla \Theta_{k}\right) V\left[\rho_{n}\right] d x=\int_{\mathbb{R}^{2}}\left(\nabla V[f] \cdot \nabla \Theta_{k}\right) V[\rho] d x, \\
& \lim _{n \rightarrow \infty} \int_{\mathbb{R}^{2}}\left(\nabla V[f] \cdot \nabla V\left[\rho_{n}\right]\right) \Theta_{k} d x=\int_{\mathbb{R}^{2}}(\nabla V[f] \cdot \nabla V[\rho]) \Theta_{k} d x .
\end{aligned}
$$


Hence for all $k \in \mathbb{N}$,

$\int_{\mathbb{R}^{2}}(f V[\rho]) \Theta_{k} d x=\int_{\mathbb{R}^{2}}\left(\nabla V[f] \cdot \nabla \Theta_{k}\right) V[\rho] d x+\int_{\mathbb{R}^{2}}(\nabla V[f] \cdot \nabla V[\rho]) \Theta_{k} d x$

Now we consider the limit $k \rightarrow \infty$. Since $f V[\rho],\left(\nabla V[f] \cdot \nabla V\left[\rho_{n}\right]\right) \in L^{1}\left(\mathbb{R}^{d}\right)$ and since $\lim _{k \rightarrow \infty} \Theta_{k}(x)=1$ for all $x \in \mathbb{R}^{d}$ with $0 \leq \Theta_{k} \leq 1$ for all $k \in \mathbb{N}$, we have

$$
\begin{gathered}
\lim _{k \rightarrow \infty} \int_{\mathbb{R}^{2}}(f V[\rho]) \Theta_{k} d x=\int_{\mathbb{R}^{2}} f V[\rho] d x, \\
\lim _{k \rightarrow \infty} \int_{\mathbb{R}^{2}}(\nabla V[f] \cdot \nabla V[\rho]) \Theta_{k} d x=\int_{\mathbb{R}^{2}} \nabla V[f] \cdot \nabla V[\rho] d x .
\end{gathered}
$$

Furthermore, for all $k \in \mathbb{N}$ and for all $x \in \mathbb{R}^{d}$,

$$
\left|\nabla \Theta_{k}(x)\right| \leq \frac{\sup _{s \in[0,1]}\left|\Theta^{\prime}(s)\right|}{k}=: \frac{K_{11}}{k} .
$$

Now we have for all $k \in \mathbb{N}$,

$$
\begin{gathered}
\left|\int_{\mathbb{R}^{2}}\left(\nabla V[f] \cdot \nabla \Theta_{k}\right) V[\rho] d x\right|=\left|\int_{\{k \leq|x| \leq 2 k\}}\left(\nabla V[f] \cdot \nabla \Theta_{k}\right) V[\rho] d x\right| \\
\leq \int_{\{k \leq|x| \leq 2 k\}}|\nabla V[f]|\left|\nabla \Theta_{k}\right||V[\rho]| d x \\
\leq \frac{K_{11}}{k}\|V[\rho]\|_{L^{\infty}\left(\mathbb{R}^{2}\right)} \sqrt{3 k^{2} \pi}\left(\int_{\{k \leq|x| \leq 2 k\}}|\nabla V[f]|^{2} d x\right)^{\frac{1}{2}} \\
\leq 4 K_{11}\|V[\rho]\|_{L^{\infty}\left(\mathbb{R}^{2}\right)}\left(\int_{\{k \leq|x| \leq 2 k\}}|\nabla V[f]|^{2} d x\right)^{\frac{1}{2}} .
\end{gathered}
$$

Due to $|\nabla V[f]| \in L^{2}\left(\mathbb{R}^{2}: \mathbb{R}^{2}\right)$,

$$
\lim _{k \rightarrow \infty} \int_{\{k \leq|x| \leq 2 k\}}|\nabla V[f]|^{2} d x=0 .
$$

We deduce from (37),

$$
\lim _{k \rightarrow \infty} \int_{\mathbb{R}^{2}}\left(\nabla V[f] \cdot \nabla \Theta_{k}\right) V[\rho] d x=0 .
$$


Employing (35), (36), (38) we deduce from (34) by passing to the limit $k \rightarrow$ $\infty$,

$$
\int_{\mathbb{R}^{2}} f V[\rho] d x=\int_{\mathbb{R}^{2}} \nabla V[f] \cdot \nabla V[\rho] d x
$$

Finally, let us verify V.3. Let $\phi \in L^{\infty}\left(\mathbb{R}^{2}\right)$ be compactly supported, let's say $\operatorname{supp}(\phi) \subseteq\{|y| \leq R\}$ for some positive $R$. Then it is easy to deduce for all $x \in \mathbb{R}^{2}$,

$$
\left|\int_{\mathbb{R}^{2}} K(x-y) \phi(y) d y\right| \leq\|\phi\|_{L^{\infty}\left(\mathbb{R}^{2}\right)} \int_{\{|y| \leq R\}}|K(y)| d y \leq K_{R}\|\phi\|_{L^{\infty}\left(\mathbb{R}^{2}\right)},
$$

where $K_{R}$ is a positive number only depending on $R$. As a consequence, $K \star \phi \in L^{\infty}\left(\mathbb{R}^{2}\right)$. Now let $\left(f_{n}\right)_{n \in N}$ be a sequence in $L^{1}\left(\mathbb{R}^{2}\right)$ with $f_{n} \rightarrow f$ weakly in $L^{1}\left(\mathbb{R}^{2}\right)$ as $n \rightarrow \infty$. Then for all $\phi \in L^{\infty}\left(\mathbb{R}^{2}\right)$ with compact support,

$$
\lim _{n \rightarrow \infty} \int_{\mathbb{R}^{2}} f_{n}(y)(K \star \phi)(y) d y=\int_{\mathbb{R}^{2}} f(y)(K \star \phi)(y) d y,
$$

while on the other hand for all $n \in \mathbb{N}$ by the Fubini-Tonelli Theorem,

$$
\begin{aligned}
& \int_{\mathbb{R}^{2}} V\left[f_{n}\right] \phi d x=-\int_{\mathbb{R}^{2}}\left(\int_{\mathbb{R}^{2}} K(x-y) f(y) d y\right) \phi(x) d x \\
& =-\int_{\mathbb{R}^{2}}\left(\int_{\mathbb{R}^{2}} K(x-y) \phi(x) d x\right) f(y) d y=-\int_{\mathbb{R}^{2}} f_{n}(y)(K \star \phi)(y) d y,
\end{aligned}
$$

and

$$
\int_{\mathbb{R}^{2}} V[f] \phi d x=-\int_{\mathbb{R}^{2}} f(y)(K \star \phi)(y) d y
$$

as well.

Example $3 \Omega=\mathbb{R}^{d}, d \geq 3$. We set $\operatorname{dom}(V):=L^{1}\left(\mathbb{R}^{d}\right)$. The Green's function of the Laplace operator is

$$
K(x):=\frac{1}{\omega_{d}(d-2)|x|^{d-2}}, \quad x \in \mathbb{R}^{d}, x \neq 0,
$$

where $\omega_{d}$ is the $(d-1)$-dimensional surface measure of the unit sphere in $\mathbb{R}^{d}$. Since $K \in L^{1}\left(\mathbb{R}^{d}\right)+L^{\infty}\left(\mathbb{R}^{d}\right)$ we have for each $f \in L^{1}\left(\mathbb{R}^{d}\right)$,

$$
K \star f=\int_{\mathbb{R}^{d}} K(.-y) f(y) d y \in L^{1}\left(\mathbb{R}^{d}\right)+L^{\infty}\left(\mathbb{R}^{d}\right) .
$$


For $f \in L^{1}\left(\mathbb{R}^{d}\right)$ we set

$$
V[f]:=-K \star f .
$$

$V[$.$] certainly satisfies \mathbf{V . 1}$.

We observe for all $i=1, \ldots, d$,

$$
\partial_{i} V[f]=-\left(\partial_{i} K\right) \star f, \quad \partial_{i} K(x)=-\frac{1}{\omega_{d}|x|^{d-1}} \frac{x_{i}}{|x|},
$$

in the sense of distributions. Furthermore, for each $i=1, \ldots, d$, the function $\partial_{i} K$ belongs to $L^{1}\left(\mathbb{R}^{d}\right)+L^{\infty}\left(\mathbb{R}^{d}\right)$.

Now let $\rho \in L^{\infty}\left(\mathbb{R}^{d}\right)$ be compactly supported ${ }^{1}$. We take $R \in(0, \infty)$ with $\operatorname{supp}(\rho) \subseteq\{|y| \leq R\}$. Then $\rho \in L^{p}\left(\mathbb{R}^{d}\right)$ for all $p \in[1, \infty]$ and we calculate for all $i=1, \ldots, d$, for all $x \in \mathbb{R}^{d}$ and for all $q \in\left(1, \frac{d}{d-1}\right), p=\frac{q}{q-1}$,

$$
\begin{gathered}
\left|\partial_{i} V[\rho](x)\right|=\frac{1}{\omega_{d}}\left|\int_{\mathbb{R}^{d}}\right| x-\left.y\right|^{1-d} \frac{x_{i}-y i}{|x-y|} \rho(y) d y\left|\leq \frac{1}{\omega_{d}} \int_{\mathbb{R}^{d}}\right| x-\left.y\right|^{1-d}|\rho(y)| d y \\
=\frac{1}{\omega_{d}} \int_{|y| \leq R}|x-y|^{1-d}|\rho(y)| d y \leq \frac{1}{\omega_{d}}\left(\int_{|y| \leq R}|x-y|^{q(1-d)} d y\right)^{1 / q}\|\rho\|_{L^{p}\left(\mathbb{R}^{d}\right)} \\
\leq \frac{\|\rho\|_{L^{p}\left(\mathbb{R}^{d}\right)}}{\omega_{d}}\left(\int_{|y| \leq R}|y|^{q(1-d)} d y\right)^{1 / q}=\frac{\|\rho\|_{L^{p}\left(\mathbb{R}^{d}\right)}}{\omega_{d}^{1 / p}}\left(\int_{0}^{R} s^{(d-1)(1-q)} d s\right)^{1 / q} \\
=\frac{R^{1-d+(d / q)}}{(d+q-d q)^{1 / q} \omega_{d}^{1 / p}}\|\rho\|_{L^{p}\left(\mathbb{R}^{d}\right)}, \quad \text { (39) }
\end{gathered}
$$

and the estimate $\left|\partial_{i} V[\rho](x)\right| \leq R\|\rho\|_{L^{\infty}\left(\mathbb{R}^{d}\right)}$ follows in analogy.

On the other hand, we have for all $x \in \mathbb{R}^{d}$ with $|x|>2 R$,

$$
\begin{aligned}
& \left|\partial_{i} V[\rho](x)\right| \leq \frac{1}{\omega_{d}}\left(\int_{|y| \leq R}|x-y|^{q(1-d)} d y\right)^{1 / q}\|\rho\|_{L^{p}\left(\mathbb{R}^{d}\right)} \\
\leq & \frac{(|x|-R)^{1-d}}{\omega_{d}}\left(\int_{|y| \leq R} 1 d y\right)^{1 / q}\|\rho\|_{L^{p}\left(\mathbb{R}^{d}\right)}=\frac{R^{d / q}}{d^{1 / q} \omega_{d}^{1 / p}}(|x|-R)^{1-d}\|\rho\|_{L^{p}\left(\mathbb{R}^{d}\right)},
\end{aligned}
$$

and the estimate $\left|\partial_{i} V[\rho](x)\right| \leq \frac{R^{d}}{d}(|x|-R)^{1-d}\|\rho\|_{L^{\infty}\left(\mathbb{R}^{d}\right)},|x|>R$, follows in analogy.

\footnotetext{
${ }^{1} \int_{\mathbb{R}^{d}} \rho d x=0$ is not required here.
} 
We set for $p \in(d, \infty)$,

$$
K(d, p, R):=\left\{\begin{array}{ll}
\omega_{d}^{-1 / p} \max \left\{\frac{(p-1)^{1-\frac{1}{p}} R^{1-\frac{d}{p}}}{(p-d)^{1-\frac{1}{p}}}, \frac{R^{\left(d-\frac{d}{p}\right.}}{d^{1-\frac{1}{p}}}\right\} & , p \in(d, \infty) \\
\max \left\{R, \frac{R^{d}}{d}\right\} & , p=\infty
\end{array} .\right.
$$

Furthermore, we put

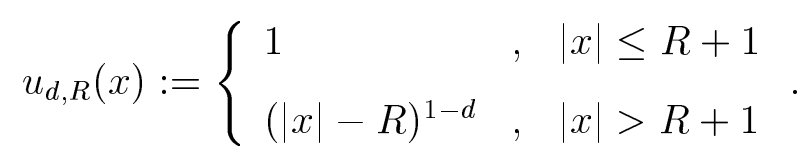

Then we deduce from (39), (40):

$$
\left|\partial_{i} V[\rho]\right| \leq K(d, p, R)\|\rho\|_{L^{p}\left(\mathbb{R}^{d}\right)} u_{d, R}, \quad p \in(d, \infty] .
$$

Since $u_{d, R} \in L^{r}\left(\mathbb{R}^{d}\right)$ for all $r \in(d /(d-1), \infty]$, we deduce from (41):

$$
\forall p \in(d, \infty], \forall r \in(d /(d-1), \infty]:
$$

There is a constant $K_{4}(d, r, p, R) \in(0, \infty)$ such that

$$
\|\nabla V[\rho]\|_{L^{r}\left(\mathbb{R}^{d}: \mathbb{R}^{d}\right)} \leq K_{4}\|\rho\|_{L^{p}\left(\mathbb{R}^{d}\right)} .
$$

In particular: $\nabla V[\rho] \in L^{2}\left(\mathbb{R}^{d}: \mathbb{R}^{d}\right)$. Hence $\rho \in \operatorname{dom}_{2}(V)$.

Remark 4. In a similiar way one can prove the estimate

$$
|V[\rho]| \leq K_{1}(d, p, R)\|\rho\|_{L^{p}\left(\mathbb{R}^{d}\right)} v_{d, R}
$$

where

$$
p \in(d / 2, \infty], \quad \rho \in L^{\infty}\left(\mathbb{R}^{d}\right), \operatorname{supp}(\rho) \subseteq\{|y| \leq R\}
$$

and

$$
K_{1}(d, p, R):= \begin{cases}\frac{1}{(d-2) \omega_{d}^{1 / p} \max \left\{\frac{(p-1)^{1-\frac{1}{p}} R^{2-\frac{d}{p}}}{(2 p-d)^{1-\frac{1}{p}}}, \frac{R^{d-\frac{d}{p}}}{d^{1-\frac{1}{p}}}\right\}}, \quad p \in\left(\frac{d}{2}, \infty\right) \\ \frac{1}{d-2} \max \left\{\frac{R^{2}}{2}, \frac{R^{d}}{d}\right\} & , p=\infty\end{cases}
$$




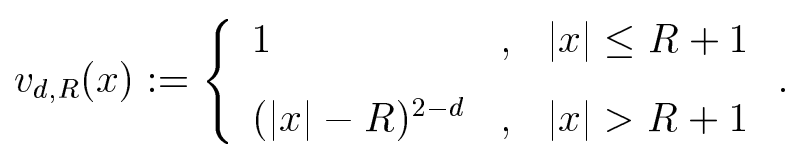

Then one can proceed as above to derive from (43) that $V[\rho] \in L^{r}\left(\mathbb{R}^{d}\right)$ for all $r \in\left(\frac{d}{d-2}, \infty\right]$ and furthermore

$$
\forall p \in(d / 2, \infty], \forall r \in(d /(d-2), \infty]:
$$

There is a constant $K_{5}(d, r, p, R) \in(0, \infty)$ such that

$$
\|V[\rho]\|_{L^{r}\left(\mathbb{R}^{d}\right)} \leq K_{5}\|\rho\|_{L^{p}\left(\mathbb{R}^{d}\right)} .
$$

The estimates (41), (42), (43), (44) allow to proceed similiar as for $d=2$. Let $f \in \operatorname{dom}_{2}(V)$ and let $\rho \in L^{\infty}\left(\mathbb{R}^{d}\right)$ be compactly supported. Then the function $(x, y) \mapsto K(x-y) f(y) \rho(x)$ belongs to $L^{1}\left(\mathbb{R}^{d} \times \mathbb{R}^{d}\right)$. We proceed as for $d=2$ with the aid of the Fubini-Tonelli Theorem to conclude

$$
\int_{\mathbb{R}^{d}} V[f] \rho d x=\int_{\mathbb{R}^{d}} V[\rho] f d x
$$

and it remains to prove

$$
\int_{\mathbb{R}^{d}} \nabla V[f] \cdot \nabla V[\rho] d x=\int_{\mathbb{R}^{d}} V[\rho] f d x .
$$

We take a sequence $\left(\rho_{n}\right)_{n \in \mathbb{N}}$ in $C_{c}^{\infty}\left(\mathbb{R}^{d}\right)$ with $\lim _{n \rightarrow \infty}\left\|\rho-\rho_{n}\right\|_{L^{2 d}\left(\mathbb{R}^{d}\right)}=0$, see [Ada]. We can assume: There is $R \in(0, \infty)$ such that $\operatorname{supp}(\rho) \subseteq\{|y| \leq R\}$ and $\operatorname{supp}\left(\rho_{n}\right) \subseteq\{|y| \leq R\}$ for all $n \in \mathbb{N}$. We deduce from (41):

$$
\nabla V\left[\rho_{n}\right] \rightarrow \nabla V[\rho] \quad \text { strongly in } L^{2}\left(\mathbb{R}^{d}: \mathbb{R}^{d}\right) \text { as } n \rightarrow \infty .
$$

Furthermore, since $K \in L_{\text {loc }}^{1}\left(\mathbb{R}^{d}\right)$ and since $\rho_{n} \in C_{c}^{\infty}\left(\mathbb{R}^{d}\right)$, we have $V\left[\rho_{n}\right] \in$ $C^{\infty}\left(\mathbb{R}^{d}\right)$ for all $n \in \mathbb{N}$, see e.g. [Ada].

Let us take $\Theta \in C^{\infty}(\mathbb{R})$ with $\Theta=1$ on $(-\infty, 0]$ and $\Theta=0$ on $[1, \infty)$. For $k \in \mathbb{N}$ we introduce ${ }^{2}$

$$
\Theta_{k}: \mathbb{R}^{d} \rightarrow \mathbb{R}, \quad \Theta_{k}(x)=\Theta(|x|-k) .
$$

\footnotetext{
${ }^{2}$ The function $\Theta_{k}$ is different from the corresponding function for $d=2$.
} 
Then $\Theta_{k} \in C_{c}^{\infty}\left(\mathbb{R}^{d}\right)$ for all $k \in \mathbb{N}$, hence $\Theta_{k} V\left[\rho_{n}\right] \in C_{c}^{\infty}\left(\mathbb{R}^{d}\right)$ for all $n, k \in \mathbb{N}$. We calculate for all $n, k \in \mathbb{N}$,

$$
\begin{gathered}
\int_{\mathbb{R}^{d}} f\left(\Theta_{k} V\left[\rho_{n}\right]\right) d x=-\int_{\mathbb{R}^{d}} V[f] \Delta\left(\Theta_{k} V\left[\rho_{n}\right]\right) d x=\int_{\mathbb{R}^{d}} \nabla V[f] \cdot \nabla\left(\Theta_{k} V\left[\rho_{n}\right]\right) d x \\
=\int_{\mathbb{R}^{d}}\left(\nabla V[f] \cdot \nabla \Theta_{k}\right) V\left[\rho_{n}\right] d x+\int_{\mathbb{R}^{d}}\left(\nabla V[f] \cdot \nabla V\left[\rho_{n}\right]\right) \Theta_{k} d x,
\end{gathered}
$$

where made use of $V\left[\rho_{n}\right] \in L^{\infty}\left(\mathbb{R}^{d}\right)$ and $\nabla V\left[\rho_{n}\right] \in L^{2}\left(\mathbb{R}^{d}: \mathbb{R}^{d}\right)$ for all $n \in \mathbb{N}$.

We consider the limit $n \rightarrow \infty$ now.

Due to (44) the sequence $\left(V\left[\rho_{n}\right]\right)_{n \in \mathbb{N}}$ is bounded in $L^{\infty}\left(\mathbb{R}^{d}\right)$ and converges strongly in $L^{r}\left(\mathbb{R}^{d}\right), r \in\left(\frac{d}{d-2}, \infty\right)$ to $V[\rho]$ as $n \rightarrow \infty$. Hence for all $k \in \mathbb{N}$,

$$
\begin{aligned}
\lim _{n \rightarrow \infty} \int_{\mathbb{R}^{d}} f\left(\Theta_{k} V\left[\rho_{n}\right]\right) d x & =\lim _{n \rightarrow \infty} \int_{\{k \leq|x| \leq k+1\}} f\left(\Theta_{k} V\left[\rho_{n}\right]\right) d x \\
= & \int_{\{k \leq|x| \leq k+1\}} f\left(\Theta_{k} V[\rho]\right) d x=\int_{\mathbb{R}^{d}} f\left(\Theta_{k} V[\rho]\right) d x .
\end{aligned}
$$

Similiar argumentations (in particular exploiting the fact that each integration is in fact an integration over a fixed (i.e. independent of $n \in \mathbb{N}$ ) bounded domain) yield for all $k \in \mathbb{N}$,

$$
\begin{aligned}
& \lim _{n \rightarrow \infty} \int_{\mathbb{R}^{d}}\left(\nabla V[f] \cdot \nabla \Theta_{k}\right) V\left[\rho_{n}\right] d x=\int_{\mathbb{R}^{d}}\left(\nabla V[f] \cdot \nabla \Theta_{k}\right) V[\rho] d x, \\
& \lim _{n \rightarrow \infty} \int_{\mathbb{R}^{d}}\left(\nabla V[f] \cdot \nabla V\left[\rho_{n}\right]\right) \Theta_{k} d x=\int_{\mathbb{R}^{d}}(\nabla V[f] \cdot \nabla V[\rho]) \Theta_{k} d x .
\end{aligned}
$$

Hence for all $k \in \mathbb{N}$,

$\int_{\mathbb{R}^{d}}(f V[\rho]) \Theta_{k} d x=\int_{\mathbb{R}^{d}}\left(\nabla V[f] \cdot \nabla \Theta_{k}\right) V[\rho] d x+\int_{\mathbb{R}^{d}}(\nabla V[f] \cdot \nabla V[\rho]) \Theta_{k} d x$

Now we consider the limit $k \rightarrow \infty$. Since $f V[\rho],\left(\nabla V[f] \cdot \nabla V\left[\rho_{n}\right]\right) \in L^{1}\left(\mathbb{R}^{d}\right)$ and since $\lim _{k \rightarrow \infty} \Theta_{k}(x)=1$ for all $x \in \mathbb{R}^{d}$ with $0 \leq \Theta_{k} \leq 1$ for all $k \in \mathbb{N}$, we have

$$
\lim _{k \rightarrow \infty} \int_{\mathbb{R}^{d}}(f V[\rho]) \Theta_{k} d x=\int_{\mathbb{R}^{d}} f V[\rho] d x
$$




$$
\lim _{k \rightarrow \infty} \int_{\mathbb{R}^{d}}(\nabla V[f] \cdot \nabla V[\rho]) \Theta_{k} d x=\int_{\mathbb{R}^{d}} \nabla V[f] \cdot \nabla V[\rho] d x .
$$

Furthermore, for all $k \in \mathbb{N}$ and for all $x \in \mathbb{R}^{d}$,

$$
\left|\nabla \Theta_{k}(x)\right| \leq \sup _{s \in[0,1]}\left|\Theta^{\prime}(s)\right|=: K_{2} .
$$

Now we have for all $k \in \mathbb{N}$,

$$
\begin{gathered}
\left|\int_{\mathbb{R}^{d}}\left(\nabla V[f] \cdot \nabla \Theta_{k}\right) V[\rho] d x\right|=\left|\int_{\{k \leq|x| \leq k+1\}}\left(\nabla V[f] \cdot \nabla \Theta_{k}\right) V[\rho] d x\right| \\
\leq \int_{\{k \leq|x| \leq k+1\}}|\nabla V[f]|\left|\nabla \Theta_{k}\right||V[\rho]| d x \\
\quad \leq K_{2}\left(\int_{\{k \leq|x| \leq k+1\}}|\nabla V[f]|^{2} d x\right)^{\frac{1}{2}}\left(\int_{\{k \leq|x| \leq k+1\}}|V[\rho]|^{2} d x\right)^{\frac{1}{2}} .
\end{gathered}
$$

If we take $R \in(0, \infty)$ with $\operatorname{supp}(\rho) \subseteq\{|y| \leq R\}$, then we have due to (43) for all $k \in \mathbb{N}$ with $k>\max \{2 R, R+1\}$,

$$
\begin{gathered}
\int_{\{k \leq|x| \leq k+1\}}|V[\rho]|^{2} d x \leq K_{1}(d, \infty, R)\|\rho\|_{\infty} \int_{\{k \leq|x| \leq k+1\}}(|x|-R)^{2-d} d x \\
=\omega_{d} K_{1}(d, \infty, R)\|\rho\|_{\infty} \int_{k}^{k+1}(s-R)^{4-2 d} s^{d-1} d s \\
\leq 2^{2 d-4} \omega_{d} K_{1}(d, \infty, R)\|\rho\|_{\infty} \int_{k}^{k+1} s^{3-d} d s \\
\leq 2^{2 d-4} \omega_{d} K_{1}(d, \infty, R)\|\rho\|_{\infty},
\end{gathered}
$$

while on the other hand due to $|\nabla V[f]| \in L^{2}\left(\mathbb{R}^{d}\right)$,

$$
\lim _{k \rightarrow \infty} \int_{\{k \leq|x| \leq k+1\}}|\nabla V[f]|^{2} d x=0 .
$$

We deduce from (49) and from (50),

$$
\lim _{k \rightarrow \infty} \int_{\mathbb{R}^{d}}\left(\nabla V[f] \cdot \nabla \Theta_{k}\right) V[\rho] d x=0 .
$$


Employing (47), (48), (51) we deduce from (46) by passing to the limit $k \rightarrow$ $\infty$,

$$
\int_{\mathbb{R}^{d}} f V[\rho] d x=\int_{\mathbb{R}^{d}} \nabla V[f] \cdot \nabla V[\rho] d x .
$$

The verification of $\mathbf{V . 3}$ can be performed in analogy to $d=2$.

Example $4 \Omega=\left\{x \in \mathbb{R}^{d}: x_{1}>0\right\}, d \in \mathbb{N}$. In this case we consider for $f \in L^{1}(\Omega)$ the Poisson equation

$$
-\Delta V=f^{*}, \quad \text { in } \mathbb{R}^{d},
$$

where for $\left(x_{1}, \ldots, x_{d}\right) \in \mathbb{R}^{d}$,

$$
f^{*}\left(x_{1}, \ldots, x_{d}\right)= \begin{cases}f\left(x_{1}, \ldots, x_{d}\right) & , x_{1}>0 \\ f\left(-x_{1}, x_{2}, \ldots, x_{d}\right) & , x_{1}<0 \\ 0 & , \text { else }\end{cases}
$$

Then we set $V[f]:=V_{\mathbb{R}^{d}}\left[f^{*}\right]\left\lfloor\Omega\right.$, where $V_{\mathbb{R}^{d}}[$.$] is the solution operator of (52)$ as discussed in the previous examples. The verification of V.1, V.2, V.3 is straight-forward.

For $\lambda>0$, the equation

$$
\lambda \Delta \Phi=n-p-C
$$

with $n=g(\alpha[\Phi]+\Phi)$ and $p=g(\beta[\Phi]-\Phi)$ has no solution with $n$ and $p$ in $L^{1}(\Omega)$ if $g>0$ on $\mathbb{R}$ (i.e. if $\underline{h}=-\infty$ ). In that case it is essential to introduce a confinement by an external potential $W$, see [BDM99], i.e. one has to replace (19) - (24) by

$$
\begin{aligned}
\lambda \Delta \Phi & =n[\Phi]-p[\Phi]-C(x), \quad x \in \Omega, \\
n[\Phi] & =g(\alpha[\Phi]+W+\Phi), \\
p[\Phi] & =g(\beta[\Phi]+W-\Phi), \\
\int_{\Omega} n[\Phi] d x & =\int_{\Omega} g(\alpha[\Phi]+\Phi-W) d x=N, \\
\int p[\Phi] d x & =\int_{\Omega} g(\beta[\Phi]-\Phi-W) d x=P,
\end{aligned}
$$

with $W \rightarrow \infty$ as $|x| \rightarrow \infty$. 
Remark 5. One may ask about the physical interpretation of $W$, in particular whether $W$ can be viewed as electrostatic potential. The problem with two species of particles with opposite sign charges is that an external electrostatic potential cannot be confining for both species, except if $W=|x|^{2}$ (by rescaling), but it is then not possible to have a charge density $C$ unless it is concentrated at the origin, see [BDM99]. The physical problem is then the question of the free expansion of two species of particles with opposite charges but same total charge, which has already been studied (for the whole space case) in [BDM99]. We shall therefore keep in mind that if one only wishes to consider electrostatic models of $W$, then for $\underline{h}=-\infty$, the only realistic cases correspond to $W=|x|^{2}$ when $\Omega$ is a cone and $C \equiv 0$, or either $N=0$ or $P=0$ and $W$ confining.

We proceed as in the previous sections to analyze (19)-(22), (56), (57) by means of the functional $E_{\lambda}$, formally defined as

$$
\begin{aligned}
& E_{\lambda}(n, p)=E^{a}(n, p)+\frac{1}{\lambda} E^{e l}(n, p), \\
& E^{a}(n, p):=\int_{\Omega} H(n) d x+\int_{\Omega} H(p) d x+\int_{\Omega}(n+p) W d x, \\
& E^{e l}(n, p):=\frac{1}{2} \int_{\Omega}|\nabla V[n-p-C]|^{2} d x,
\end{aligned}
$$

where $(n, p) \in \mathcal{C}$, i.e. $n, p \in L_{+}^{1}(\Omega)$ with $\|n\|_{L^{1}(\Omega)}=N$ and $\|p\|_{L^{1}(\Omega)}=P$. As in the previous sections we set the electrostatic energy $E^{e l}(n, p)$ equal to $\infty$ whenever $n-p-C \notin \operatorname{dom}_{2}(V)$. The confining potential $W$ of (58) is assumed to be bounded below. Hence the last integral of $E^{a}(n, p)$ has a well-defined value in $\mathbb{R} \cup\{\infty\}$. A bit more delicate is the integrability of $H(n), H(p)$. In contrast to the situation for bounded domains, the convexity of $H$ is not sufficient to assign to each of the first two integrals of $E^{a}(n, p)$ a value in $\mathbb{R} \cup\{\infty\}$. In fact, we have to impose an additional assumption included in the following

Proposition 10. Let $\Omega \subseteq \mathbb{R}^{d}, d \in \mathbb{N}$, be a nonvoid domain. Let $N, P \geq 0$ and let $W \in L_{l o c}^{1}(\Omega)$ be bounded below. Furthermore, assume A.2 - A.5, V.1 - V.3 and

A.6 There are $\mu, \nu \in \mathbb{R}$ with $n_{N}:=g(\mu-W), p_{P}:=g(\nu-W) \in L^{1}(\Omega)$,

$$
\int_{\Omega} n_{N} d x=N, \quad \int_{\Omega} p_{P} d x=P
$$


and $H^{-}\left(n_{N}\right), H^{-}\left(p_{P}\right) \in L^{1}(\mathbb{R})$.

Then $E_{\lambda}$ is bounded below on $\mathcal{C}$.

Proof. We exploit the convexity of $H$. We have for each $n \in L_{+}^{1}(\Omega)$ with $\int_{\Omega} n d x=N$

$$
\begin{aligned}
&((H(n)+n W)-(\left.\left.H\left(n_{N}\right)+n_{N} W\right)\right)=H(n)-H\left(n_{N}\right)+W\left(n-n_{N}\right) \\
& \geq H^{\prime}\left(n_{N}\right)\left(n-n_{N}\right)+W\left(n-n_{N}\right) \\
&=\left(h\left(n_{N}\right)+W\right)\left(n-n_{N}\right) \geq \mu\left(n-n_{N}\right),
\end{aligned}
$$

because $h\left(n_{N}\right)=h(g(\mu-W))=\mu-W$ whenever $n_{N}>0$ and $h\left(n_{N}\right)(n-$ $\left.n_{N}\right)=h\left(n_{N}\right) n \geq(\mu-W) n=(\mu-W)\left(n-n_{N}\right)$ whenever $n_{N}=0$. Hence,

$$
\begin{aligned}
& \int_{\Omega}(H(n)+n W) d x-\int_{\Omega}\left(H\left(n_{N}\right)+n_{N} W\right) d x \\
& \geq \int_{\Omega} \mu\left(n-n_{N}\right) d x=\mu\left(\int_{\Omega} n d x-\int_{\Omega} n_{N} d x\right)=\mu(M-M)=0
\end{aligned}
$$

and the inequality

$$
\int_{\Omega}(H(p)+p W) d x-\int_{\Omega}\left(H\left(p_{P}\right)+p_{P} W\right) d x \geq 0
$$

with $p \in L_{+}^{1}(\Omega)$ with $\int_{\Omega} p d x=P$ follows analogously. Due to the assumuption $H^{-}\left(n_{N}\right), H^{-}\left(p_{P}\right) \in L^{1}(\Omega)$ and due to the assumed boundedness of $W$ from below we deduce that $\int_{\Omega}\left(H\left(n_{N}\right)+n_{N} W\right) d x, \int_{\Omega}\left(H\left(p_{P}\right)+p_{P} W\right) d x$ have values in $\mathbb{R} \cup\{\infty\}$.

Remark 6. a) Since $g$ is strictly increasing there is at most one pair $(\mu, \nu) \in$ $\mathbb{R}^{2}$ satisfying A.6.

b) If $N=0$ and if $g>0$ on $\mathbb{R}$ (i.e. if and only if $\underline{h}=-\infty$ ), then there is no $\mu \in \mathbb{R}$ with $\int_{\Omega} g(\mu-W) d x=N=0$. Hence, if one wishes to consider the case $N=0$ with $\underline{h}=-\infty$, then one has to modify the functional $E_{\lambda}$ by cancelling (or setting to zero, respectively) all terms which involve $n$. We leave the details of the corresponding analysis to the reader.

c) Proposition 10 does not exclude $E_{\lambda} \equiv \infty$ on $\mathcal{C}$.

Now let us turn our attention to the existence of minimizers of $E_{\lambda}$ in $\mathcal{C}$. As an example, we state the following result in case of $\bar{h}=+\infty$ for which a purely variational argument provides an immediate answer. 
Theorem 11. Let $\Omega \subseteq \mathbb{R}^{d}, d \in \mathbb{N}$, be a nonvoid domain. Let $N, P>0$ and let $W \in L_{l o c}^{1}(\Omega)$. Furthermore, assume A.2 - A.6, V.1 - V.3 and

1. $C \in L^{1}(\Omega)$.

2. $W$ is bounded below. $\liminf _{x \in \Omega,|x| \rightarrow \infty} W(x)=\infty$.

3. $\bar{h}=\infty$.

4. $E^{a}+E_{\text {el }} \not \equiv \infty$ on $\mathcal{C}$.

5. $E^{a}\left(n_{N}, p_{P}\right)<\infty$ (that is, $\left.H\left(n_{N}\right)+H\left(p_{P}\right)+\left(n_{N}+p_{P}\right) W \in L^{1}(\Omega)\right)$, where $n_{N}, p_{P}$ are as in A.6.

Then $E_{\lambda}$ has a unique minimizer $\left(n_{0}, p_{0}\right)$ in $\mathcal{C}$ and the triple $\left(\Phi_{0}, n_{0}, p_{0}\right)=$ $\left(-\lambda^{-1} V_{0}, n_{0}, p_{0}\right)$ with $V_{0}=V\left[n_{0}-p_{0}-C\right]$ is a solution (the unique "equilibrium solution") of (53)-(57).

Remark 7. a) Assumption 4. $E_{\lambda} \not \equiv \infty$ on $\mathcal{C}$ implies in case of $\Omega=\mathbb{R}^{1}$, $\Omega=\mathbb{R}^{2}$ or in case of $\Omega \subset \mathbb{R}^{d}, d \in \mathbb{N}$, with meas $(\Omega)<\infty$ global charge neutrality $N-P=\int_{\Omega} C$ which is not the case for $\Omega=\mathbb{R}^{d}, d \geq 3$

b) By straight-forward modifications one can also include $N=0, P>0$ or $N>0, P=0$.

Proof. $E_{\lambda}$ is bounded below by Proposition 10. Furthermore, $E_{\lambda} \not \equiv \infty$ on $\mathcal{C}$. Hence $\inf _{\mathcal{C}} E_{\lambda}>-\infty$. We apply a standard minimization argument. Since $E_{\lambda}$ is strictly convex, $E_{\lambda}$ has at most one minimizer. This minizer is shown to exist by taking the limit of a minimizing sequence $\left(n_{k}, p_{k}\right)_{k \in \mathbb{N}}$. The limit $\left(n_{0}, p_{0}\right)$ of this sequence belongs to $\mathcal{C}$ because of the weak- $L^{1}$ compactness of any minimizing sequence according to the Dunford-Pettis criterion: there is no concentration because $H$ is superlinear at $\infty(\bar{h}=\infty)$ and no vanishing (because of the growth of $W$ at $\infty$ ) in the language of concentrationcompactness theory.

As shown in the proof of Proposition 10, we have $H(n)+H(p)+(n+p) W \geq$ $H\left(n_{N}\right)+H\left(p_{P}\right)+\left(n_{N}+p_{P}\right) W \in L^{1}(\Omega)$ for all $(n, p) \in \mathcal{C}$. Hence the functional $E_{\lambda}^{a}$ is lower semicontinuous with respect to weak convergence in $L^{1}(\Omega)$ and we have

$$
\begin{aligned}
\int_{\Omega} H\left(n_{0}\right) & d x+\int_{\Omega} H\left(p_{0}\right) d x+\int_{\Omega} W\left(n_{0}+p_{0}\right) d x \\
& \leq \liminf _{k \rightarrow \infty} \int_{\Omega} H\left(n_{k}\right) d x+\int_{\Omega} H\left(p_{k}\right) d x+\int_{\Omega} W\left(n_{k}+p_{k}\right) d x .
\end{aligned}
$$


Furthermore, since $\left(\nabla V_{k}\right)_{k \in \mathbb{N}}, V_{k}=V\left[n_{k}-p_{k}-C\right]$, is bounded in $L^{2}(\Omega$ : $\mathbb{R}^{d}$ ), we have - maybe after extracting a subsequence but without changing notations - for all $i=1, \ldots, d$,

$$
\partial_{i} V_{k} \rightarrow Z_{i}, \quad \text { weakly in } L^{2}(\Omega) \text { as } k \rightarrow \infty,
$$

hence

$$
\int_{\Omega}\left(Z_{1}^{2}+\ldots+Z_{d}^{2}\right) d x \leq \liminf _{k \rightarrow \infty} \int_{\Omega}\left|\nabla V_{k}\right|^{2} d x .
$$

Since $C \in L^{1}(\Omega)$ we have $n_{k}-p_{k}-C \rightarrow n_{0}-p_{0}-C$ weakly in $L^{1}(\Omega)$ as $k \rightarrow \infty$. We deduce from V.3, $V_{k} \rightarrow V_{0}=V\left[n_{0}-p_{0}-C\right]$ in the sense of distributions. Hence $Z_{i}=\partial_{i} V_{0}, i=1, \ldots, d$ and we deduce $E_{\lambda}\left(n_{0}, p_{0}\right) \leq$ $\liminf _{k \rightarrow \infty} E_{\lambda}\left(n_{k}, p_{k}\right)=\inf _{\mathcal{C}} E_{\lambda}$ from (59) and (60).

Now we shall derive the associated variational inequalities. With the aid of V.2 we deduce from standard arguments,

$h\left(n_{0}\right)+W+\lambda^{-1} V_{0}=\alpha \quad$ on $\left\{n_{0}>0\right\}, \quad h\left(p_{0}\right)+W-\lambda^{-1} V_{0}=\beta \quad$ on $\left\{p_{0}>0\right\}$,

for some $\alpha, \beta \in \mathbb{R}$. In case of $\underline{h}>-\infty$ the function $H$ is differentiable on each compact subset of $[0, \infty)$ and in this case we obtain by standard arguments

$$
\underline{h}+W+\lambda^{-1} V_{0} \geq \alpha \quad \text { on }\left\{n_{0}=0\right\}, \quad \underline{h}+W-\lambda^{-1} V_{0} \geq \beta \quad \text { on }\left\{p_{0}=0\right\},
$$

and we conclude $n_{0}=g\left(\alpha-W-\lambda^{-1} V_{0}\right), p_{0}=g\left(\beta-W+\lambda^{-1} V_{0}\right)$,

i.e. $\left(-\lambda^{-1} V_{0}, n_{0}, p_{0}\right)$ is a solution of (53)-(57). It remains to consider the case $\underline{h}=-\infty$. It sufficies to prove: $n_{0}>0$ and $p_{0}>0$ almost everywhere on $\Omega$. This is shown in an indirect way. If $n_{0}=0$ on a subset $\Omega_{0}$ of $\Omega$ with meas $\left(\Omega_{0}\right)>0$, then there is a compact subset $K_{0}$ of $\Omega_{0}$ with meas $\left(K_{0}\right)>0$ as well and one can take a test function $\phi \in L^{\infty}(\Omega)$ which is compactly supported in $\bar{\Omega}, \operatorname{ran}(\phi)=\left\{c_{0},-c_{1}\right\}$ (where $c_{0}, c_{1}$ are positive real numbers), $\phi(x)=c_{0}$ if and only if $x \in K_{0}, \phi^{-1}\left(-c_{1}\right) \subseteq\left\{\varepsilon \leq n_{0} \leq \varepsilon^{-1}\right\}$ (where $\varepsilon \in(0,1)$ is apropriately chosen), and $\int_{\Omega} \phi d x=0$. We observe: $\left(n_{0}+\delta \phi, p_{0}\right) \in \mathcal{C}$ for all sufficiently small $\delta$. Since $\left(n_{0}, p_{0}\right)$ minimizes $E_{\lambda}$ in $\mathcal{C}$ one has for all sufficiently small $\delta \in(0, \infty)$,

$$
E_{\lambda}\left(n_{0}+\delta, p_{0}\right)-E_{\lambda}\left(n_{0}, p_{0}\right) \geq 0
$$

while it is not difficult to deduce from $\underline{h}=-\infty$ via $\mathbf{V} .2$ that $\lim _{\delta \rightarrow 0}\left(E_{\lambda}\left(n_{0}+\right.\right.$ $\left.\left.\delta, p_{0}\right)-E_{\lambda}\left(n_{0}, p_{0}\right)\right) / \delta=-\infty$. Hence $n_{0}>0$ almost everywhere on $\Omega . p_{0}>0$ almost everywhere on $\Omega$ follows in analogy. 
Remark 8. a) Propositon 11 highlights the role of the confining potential $W$ which ensures weak compactness of minimizing sequences of $E_{\lambda}$.

b) One may ask whether each solution $\left(\Phi_{0}, n_{0}, p_{0}\right)$ of $(19)-(22),(56),(57)$ is actually a minimizer of $E_{\lambda}$ in $\mathcal{C}$. The discussion of this (very natural) question, however, requires rather technical additional assumptions (and is therefore omitted here): In contrast to the situation for bounded domains there is no a priori estimate which ensures $E_{\lambda}\left(n_{0}, p_{0}\right)<\infty$.

The introduction of the confining potential $W$ is inevitable whenever $\underline{h}=$ $-\infty$. Let us turn our attention to a discussion of (19)-(24) (i.e. the model equations without $W$ ) in the case $\underline{h}>-\infty$ now.

In this situation it is possible to build solutions with compact support as follows (we recall: without any external confinement potential !).

Take a $\Phi \in C^{2}(\Omega)$ with compact support in $\Omega$ and consider $n_{\lambda}=g(\alpha+\Phi)$ and $p_{\lambda}=g(\beta-\Phi)$ for $\alpha$ and $\beta$ such that $\underline{h}-\alpha>0>\beta-\underline{h}$. The density $n_{\lambda}$ (respectively $p_{\lambda}$ ) is supported in the set corresponding to $\Phi>\underline{h}-\alpha$ (respectively $\Phi<\beta-\underline{h}$ ). We may then define

$$
C=-\lambda \Delta \Phi+g(\alpha+\Phi)-g(\beta-\Phi),
$$

and compute $N$ and $P$ corresponding to $\alpha, \beta$ and $\Phi$. Then the triple $\left(\Phi, n_{\lambda}, p_{\lambda}\right)$ is certainly a solution of (19)-(24). In particular, we observe that global neutrality holds due to the fact that $\Phi$ has a compact support. $N \neq 0$

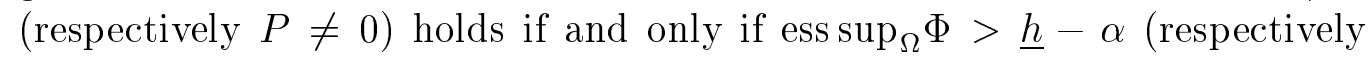
$\left.\operatorname{ess~inf}_{\Omega} \Phi<\beta-\underline{h}\right)$.

We note that this construction actually does not depend on whether $\Omega$ is bounded or not, but only on the condition $\operatorname{supp}(\Phi) \subset \subset \Omega$. The condition that $\Phi$ has a compact support can be replaced by the condition that $\Phi$ is equal to a constant $\Phi_{\infty}$ outside a compact subset of $\Omega$, with $\underline{h}-\alpha>\Phi_{\infty}>\beta-\underline{h}$. In unbounded domains, it is actually sufficient to assume that $\Phi=\Phi_{\infty}$ in a neighborhood of $\partial \Omega$ and

$$
\beta-\underline{h}<\liminf _{|x| \rightarrow \infty, x \in \Omega} \Phi(x) \leq \limsup _{|x| \rightarrow \infty, x \in \Omega} \Phi(x)<\underline{h}-\alpha .
$$

If there exists a solution $\Phi$ with $\Phi-\Phi_{\infty}$ compactly supported in $\Omega$ for a constant $\Phi_{\infty} \in(\beta-\underline{h}, \underline{h}-\alpha)$, then the global neutrality condition

$$
N-P-\int_{\Omega} C d x=0
$$


holds, but this is not necessarily the case if $\Omega$ is unbounded. Consider for instance a radial potential $\Phi$ which is asymptotically periodic, oscillating between two values in $(\beta-\underline{h}, \underline{h}-\alpha)$, with $C=-\Delta \Phi$ for $|x|$ large enough (case $\underline{h}>-\infty)$ : $C$ is clearly not in $L^{1}(\Omega)$.

The global electroneutrality does not either hold in the presence of an external potential $W$. We can for instance (see [Dol91]) consider in $\Omega=\mathbb{R}^{d}$ $(d \geq 3)$ the equation

$$
\Delta \Phi=N \frac{e^{\Phi-W(x)}}{\int_{\Omega} e^{\Phi-W(x)} d x}-P \frac{e^{-\Phi-W(x)}}{\int_{\Omega} e^{-\Phi-W(x)} d x}
$$

with $\lim _{|x| \rightarrow+\infty} \Phi(x)=0, W(x)=\frac{1}{2}|x|^{2}, C \equiv 0$. If $N \neq P$, the global electroneutrality condition (61) is not satisfied. This example is easy to generalize to any potential $W$ such that $\liminf _{|x| \rightarrow+\infty}|x|^{d-2} W(x)=+\infty$ and $e^{-W} \in L^{1}\left(\mathbb{R}^{d}\right)$ and $C \in L^{1}\left(\mathbb{R}^{d}\right)$ (with compact support for example). More striking is the fact that we can state an existence result without global electronuetrality even in the case without confining potential $(W \equiv 0)$ provided $d \geq 3($ and $\underline{h}>-\infty)$.

\section{ACKNOWLEDGEMENT}

This research was partially supported by the grant ERBFMRXCT970157 (TMR-Network) from the EU.

\section{References}

[Ada] R.A. Adams, Sobolev Spaces, Academic Press, 1975.

[AMT98] A. Arnold, P.A. Markowich, G. Toscani, On large time asymptotics for drift-diffusion Poisson systems, Preprint ESI no. 655 (1999), Preprint TMR "Asymptotic Methods in Kinetic Theory" no. 12 (1998), submitted to Transp. Theo. Stat. Phys.

[AMTU98] A. Arnold, P.A. Markowich, G. Toscani, A. Unterreiter, On logarithmic Sobolev inequalities, Csiszár-Kullback inequalities, and the rate of convergence to equilibrium for Fokker-Planck type equations, Preprint TMR "Asymptotic Methods in Kinetic Theory" no. 1 (1998). 
[ArNi] A. Arnold, F. Nier, The Two-Dimensional Wigner-Poisson Problem for an Electron Gas in the Charge Neutral Case, Math Meth Appl Sci 14 (1991), 595-613.

[BiDo99] P. Biler, J. Dolbeault, Long time behavior of solutions to NernstPlanck and Debye-Hückel drift-diffusion systems, to appear in Annales Henri Poincaré, Preprint Ceremade no. 9915.

[BDM99] P. Biler, J. Dolbeault, P.A. Markowich, Large time asymptotics of nonlinear drift-diffusion systems with Poisson coupling, Preprint Ceremade no. 9932.

[CDMS99] L. Caffarelli, J. Dolbeault, P.A. Markowich, C. Schmeiser, On Maxwellian Equilibria of Insulated Semiconductors, Preprint Ceremade no. 9939, to appear in Interfaces and Free Boundaries.

[Caf00] L. Caffarelli, The obstacle problem, manuscript, 2000.

[CJMTU00] J.A. Carrillo, A. Jüngel, P.A. Markowich, G. Toscani, A. Unterreiter, Entropy dissipation methods for degenerate parabolic problems and generalized Sobolev inequalities, Preprint TMR "Asymptotic Methods in Kinetic Theory" no. 119 (2000).

[Dol91] J. Dolbeault, Stationary states in plasma physics: Maxwellian solutions of the Vlasov-Poisson system, Math Models Methods Appl Sci 1 (1991), 183-208.

[Gaj85] H. Gajewski, On existence, uniqueness and asymptotic behavior of solutions of the basic equations for carrier transport in semiconductors, ZAMM 65 (1085), pp 101-108.

[GaGr89] H. Gajewski, K. Gröger, Semiconductor Equations for variable Mobilities Based on Boltzmann Statistics or FERMI-DIRAC Statistics, Math Nachr 140 (1989), pp 7-36.

[Gas] I. Gasser. On the Relations between Different Macroscopic Models for Semiconductors. Habilitationsschrift Universität Hamburg, 2000.

[GLMS99] I. Gasser, D. Levermore, P.A. Markowich, C. Schmeiser, The initial time layer problem and the quasineutral limit in the semiconductor drift-diffusion model, manuscript 1999. 
[Groe86] K. Gröger, On the basis equations for carrier transport in semiconductors, J Math Anal Appl 113 (1986), pp 12-35.

[MRS90] P.A. Markowich, C. Ringhofer, C. Schmeiser, Semiconductor Equations, Springer, 1990.

[Ott00] F. Otto, The geometry of dissipative evolution equations: the porous medium equation. to appear in Comm PDE.

[Rud] W. Rudin, Real and Complex Analysis, McGraw-Hill, 1987.

[Unt97] A. Unterreiter, The thermal equilibrium solution of a generic bipolar quantum hydrodynamic model, Comm Math Phys 188 (1997), pp 6988.

[Zie89] W.P. Ziemer, Weakly differentiable functions, Springer, 1989. 